\title{
Auditory processing in the zebra finch midbrain: single unit responses and effect of rearing experience
}

\author{
Priscilla Logerot ${ }^{1}$, Paul F. Smith ${ }^{2}$, Martin Wild ${ }^{3}$, M Fabiana Kubke ${ }^{\text {Corresp. } 4}$ \\ 1 Anatomy and Medical Imaging, University of Auckland, University of Auckland, Auckland, New Zealand \\ 2 Dept. of Pharmacology and Toxicology, School of Biomedical Sciences, Brain Health Research Centre, Brain Research New Zealand, and Eisdell Moore \\ Centre, University of Otago, Dunedin, New Zealand \\ 3 Anatomy and Medical Imaging and Eisdell Moore Centre, University of Auckland, University of Auckland, Auckland, New Zealand \\ 4 Anatomy and Medical Imaging, Centre for Brain Research and Eisdell Moore Centre, University of Auckland, University of Auckland, Auckland, New \\ Zealand
}

Corresponding Author: M Fabiana Kubke

Email address: f.kubke@auckland.ac.nz

In birds the auditory system plays a key role in providing the sensory input used to discriminate between conspecific and heterospecific vocal signals. In those species that are known to learn their vocalizations, e.g., songbirds, it is generally considered that this ability arises and is manifest in the forebrain, although there is no a priori reason why brainstem components of the auditory system could not also play an important part. To test this assumption, we used groups of normal reared and cross-fostered zebra finches that had previously been shown in behavioural experiments to reduce their preference for conspecific songs subsequent to cross fostering experience with Bengalese finches, a related species with a distinctly different song. The question we asked, therefore, is whether this experiential change also changes the bias in favour of conspecific song displayed by auditory midbrain units of normally raised zebra finches. By recording the responses of single units in MLd to a variety of zebra finch and Bengalese finch songs in both normally reared and cross-fostered zebra finches, we provide a positive answer to this question. That is, the differences in responses to conspecific and heterospecific songs seen in normal reared zebra finches is reduced following cross-fostering. In birds the virtual absence of mammalian-like cortical projections upon auditory brainstem nuclei argues against the interpretation that MLd unit changes, such as those observed in the present experiments, in terms of top-down influences on sensory processing. Instead, it appears that MLd units can be influenced significantly by sensory inputs arising directly from a change in auditory experience during development. 
1 Auditory processing in the zebra finch midbrain: single unit responses and effect of rearing

2 experience.

3 Priscilla Logerot ${ }^{1}$, Paul F. Smith ${ }^{2}$, J Martin Wild ${ }^{3}$, M Fabiana Kubke ${ }^{4}$

41 Anatomy and Medical Imaging, University of Auckland, University of Auckland,

5 Auckland, New Zealand

62 Dept. of Pharmacology and Toxicology, School of Biomedical Sciences, Brain Health

7 Research Centre, Brain Research New Zealand, and Eisdell Moore Centre, University of

8 Otago, Dunedin, New Zealand

3 Anatomy and Medical Imaging and Eisdell Moore Centre, University of Auckland,

10 University of Auckland, Auckland, New Zealand

114 Anatomy and Medical Imaging, Centre for Brain Research and Eisdell Moore Centre,

12 University of Auckland, University of Auckland, Auckland, New Zealand

13

14 Corresponding Author:

M Fabiana Kubke

16

Department of Anatomy and Medical Imaging

University of Auckland

85 Park Road

Grafton, Auckland

New Zealand

f.kubke@auckland.ac.nz 


\section{Manuscript to be reviewed}

\section{ABSTRACT}

24

In birds the auditory system plays a key role in providing the sensory input used to discriminate between conspecific and heterospecific vocal signals. In those species that are known to learn their vocalizations, e.g., songbirds, it is generally considered that this ability arises and is manifest in the forebrain, although there is no a priori reason why brainstem components of the auditory system could not also play an important part. To test this assumption, we used groups of normal reared and cross-fostered zebra finches that had previously been shown in behavioural experiments to reduce their preference for conspecific songs subsequent to cross fostering experience with Bengalese finches, a related species with a distinctly different song. The question we asked, therefore, is whether this experiential change also changes the bias in favour of conspecific song displayed by auditory midbrain units of normally raised zebra finches. By recording the responses of single units in MLd to a variety of zebra finch and Bengalese finch songs in both normally reared and cross-fostered zebra finches, we provide a positive answer to this question. That is, the differences in responses to conspecific and heterospecific songs seen in normal reared zebra finches is reduced following cross-fostering. In birds the virtual absence of mammalian-like cortical projections upon auditory brainstem nuclei argues against the interpretation that MLd unit changes, such as those observed in the present experiments, in terms of top-down influences on sensory processing. Instead, it appears that MLd units can be influenced significantly by sensory inputs arising directly from a change in auditory experience during development. 


\section{Manuscript to be reviewed}

\section{INTRODUCTION}

47 In songbirds, as in many other vertebrates, vocal signals play a key role in communication (Catchpole and Slater 2003). Most vertebrates use vocal communication signals that are innate and supported by a suite of interconnected brainstem nuclei that contribute to the production and identification of conspecific calls (Kennedy 1974, Kubke and Wild 2018). A handful of lineages (including songbirds) have, in addition to the innate repertoire, vocalizations that are learned during a critical developmental period (Zann 1990, Bolhuis and Moorman 2015). In songbirds these vocalizations are learned first by memorizing the song of a tutor (usually the father) during early life, and by later learning to match their vocal output to the internal template of the song (Bolhuis and Moorman 2015). A set of forebrain nuclei collectively known as the 'song system' are, under the influence of auditory input, involved in the learning, production, and maintenance of the song (Fig. 1A) (Brainard and Doupe 2000). Most studies on auditory processing in songbirds have focused on forebrain areas; the role of the auditory brainstem in extracting auditory information used in the learning, production and maintenance of song is less well known.

\section{INSERT FIGURE 1 AROUND HERE} responses to spectral and temporal features of species-specific vocal communication signals (Scheich et al. 1977, Feng et al. 1990, Bodnar et al. 2001, Bass and McKibben 2003, Šuta et al. 2003, Bass et al. 2005, Covey and Carr 2005, Portfors and Sinex 2005, Rose and Gooler 2007, Rodríguez et al. 2009, Holmstrom et al. 2010, Wilczynski and Ryan 2010, Rose et al. 2011, Sayegh et al. 2011, Pollak 2013). In songbirds, numerous studies describe auditory processing of the learned song primarily in the auditory forebrain, where different regions are suggested to encode different aspects of the perception and selection of conspecific signals (for review see Knudsen and Gentner 2010). These nuclei receive the necessary auditory input indirectly by way of ascending inputs from the brainstem auditory nuclei and thalamus (Fig. 1B, Nottebohm et al. 1976, Nottebohm et al. 1982). Studies on the role of the lower auditory system in general, and the midbrain in particular, in the processing of innate and learned vocal signals are lacking or limited, despite the fact that the auditory 
80 midbrain of songbirds, like that of other vertebrates, is well positioned to serve as a major

81 centre where selectivity to learned vocal signals could arise (Feng et al. 1990, Bass et al.

82 2005, Covey and Carr 2005, Portfors and Sinex 2005, Rose and Gooler 2007, Wilczynski

83 and Ryan 2010, Vonderschen and Chacron 2011, Wenstrup et al. 2012).

84 The major contribution to our understanding of auditory processing in the songbird auditory brainstem has come from the work of Woolley and colleagues in the zebra finch, who showed that neurons in the auditory midbrain (mesencephalicus lateralis pars dorsalis,

87 MLd) are tuned to specific spectro-temporal modulations of the conspecific song, suggesting

88 that in birds, too, the auditory midbrain plays a central role in the processing of

89 communication signals (Woolley and Casseday 2004, Woolley et al. 2005, Woolley et al.

90 2006, Woolley et al. 2009, Schneider and Woolley 2010, Woolley et al. 2010, Woolley 2012).

91 Further, the work of Woolley and colleagues also showed that the responses found in the

92 auditory midbrain could be modified by early developmental experience, thus linking the

93 midbrain to the filtering features that support song learning (Woolley et al. 2010).

94 Woolley and colleagues (Woolley et al. 2010) exploited the changes in song structure 95 and behavioural preference that follow the cross fostering of zebra finches by Bengalese 96 finches (Campbell and Hauber 2009). When zebra finch chicks are cross-fostered by 97 Bengalese finches they grow to produce a song that: (1) incorporates elements of the foster 98 parents; (2) contains more elements than the normal zebra finch song but fewer than the 99 Bengalese finch song; and (3) is longer than the normal zebra finch song but shorter than 100 that of the foster male (Eales 1987, Clayton 1989, Takahasi et al. 2006).

101 Woolley et al. compared the responses to zebra finch and Bengalese finch songs in the 102 MLds of normal reared zebra finches, and zebra finches that had been cross-fostered by 103 Bengalese finches (Woolley et al. 2010). They report that presentation of zebra finch or 104 Bengalese finch songs in normal-reared and cross-fostered birds appear to evoke similar 105 firing rates in MLd units, even though, overall, the mean firing rate is diminished in cross106 fostered birds. Mutual information rates were also similar when comparing responses to 107 zebra finch or Bengalese finch songs in both normal-reared and cross-fostered birds. This is 108 in contrast to behavioural studies (Campbell and Hauber 2009) in which normal reared zebra 109 finches were shown to 'prefer' zebra finch song over Bengalese finch song, whereas cross110 fostered zebra finches associated equally with both types of songs. Taken together, these 111 results would suggest that the different behavioural preferences to zebra finch or Bengalese

112 finch song in normal reared and cross fostered birds cannot be accounted for by response 113 differences in MLd. 
114 Using a similar paradigm to that of Woolley et al. 2010, we here examine the responses

115 of units in MLds to zebra finch and Bengalese finch songs in normal-reared and cross

116 fostered zebra finches (Campbell and Hauber 2009, 2010). In contrast to Woolley et al.'s

117 findings, we find that units in MLd change their response properties based on developmental

118 experience. Our data indicate that units in MLd of normally-reared zebra finches show a bias

119 toward conspecific (zebra finch) song, whereas in zebra finches cross-tutored by Bengalese

120 finches, the bias of MLd units towards zebra finch vocalizations was reduced. Thus, our

121 findings suggest that the ontogenetic changes that affect behavioural preference to

122 conspecific over heterospecific song are paralleled by neuronal responses recorded from

123 MLd.

\section{METHODS}

\section{ANIMALS AND ETHICAL CONSIDERATIONS}

126 The experimental work was carried out in compliance with and approval from the

127 University of Auckland Animal Ethics Committee (R425) in accordance with the University of

128 Auckland Code of Ethical Conduct for the Use of Animals for Teaching and Research, the

129 Animal Welfare Act 1999 (New Zealand), and The National Animal Ethics Advisory

130 Committee (NAEAC) Good Practice Guide for the Use of Animals in Research, Testing and

131 Teaching. Birds were provided with ad-lib water and commercial dry finch-mix seeds

132 (Animates ${ }^{\mathrm{TM}}$ ), spray millet and additional weekly treats (fresh grasses, corn boiled egg, and

133 rice). Birds were also supplied with calcium supplements and grit.

\section{NORMAL REARED ZEBRA FINCHES}

135 Six adult zebra finches ( 2 males and 4 females $>100$ days old) that were raised having

136 been exposed only to their conspecifics were used in this study. Birds were bred in an aviary

137 at the Department of Psychology, University of Auckland (Campbell and Hauber, 2010).

138 Light was provided via a compact Arcadia fluorescent bird lamp (2.4\% UVB and 12\% UVA)

139 which imitated outdoor conditions. The mean temperature in the aviary was kept at $21 \pm 3^{\circ} \mathrm{C}$,

140 daily humidity between $35-65 \%$ and care was taken to keep a constant airflow. Pairs of

141 zebra finches (Taeniopygia guttata) were housed together in indoor cages and once the

142 chicks reached adulthood (>100 days), they were transferred to a holding indoor aviary ( $2 \mathrm{~m}$

$143 \times 1 \mathrm{~m} \times 2 \mathrm{~m}$ ) in the Faculty of Medical and Health Sciences. At all times, birds were held at a

144 constant light/dark cycle of $14 \mathrm{~h} / 10 \mathrm{~h}$ in the aviary and birds were provided with food and

145 water ad lib. Thus, the ZF-ZF group had been only exposed to conspecific song. 
147 Six male zebra finches (>100 days old) that were raised by Bengalese finches (ZF-CF)

148 were used. These zebra finches had previously been used in behavioural experiments and a

149 full description of the rearing conditions is available in (Campbell and Hauber 2009). Briefly,

150 zebra finch chicks (3-5 days old) were transferred into the nest of a Bengalese finch pair in

151 one of two outdoor aviaries in Auckland $(2 \mathrm{~m} \times 1 \mathrm{~m} \times 2 \mathrm{~m})$, and exposed to natural photoperiod

152 and weather conditions. The chicks were therefore reared hearing male and female

153 Bengalese finches and tutored by a Bengalese finch male, but were also exposed to wild

154 birds species found commonly in suburban Auckland (Blackbird Turdus merula; House

155 Sparrow Passer domesticus; Song Thrush Turdus philomelos; Starling Sturnus vulgaris; and

156 Tui Prosthermadera novaeseelandiae). After reaching adulthood ( $>100$ days), the cross-

157 fostered birds were transferred into single-sex aviaries where they were housed with

158 fostered conspecifics. After the behavioural experiments were completed, the birds were

159 finally transferred to an indoor aviary $(2 \mathrm{~m} \times 1 \mathrm{~m} \times 2 \mathrm{~m})$ at the Faculty of Medical and Health

160 Sciences, co-housed with the group of normal reared zebra finches. . At all times, birds were

161 held at a constant light/dark cycle of $14 \mathrm{~h} / 10 \mathrm{~h}$ in the aviary and birds were provided with food

162 and water ad lib. These birds did not hear normal conspecific vocalisations before

163 behavioural testing, at which time their songs were crystallised.

SURGERY

165 Birds were deeply anesthetised with an intramuscular injection of a mixture consisting of

$16655 \mathrm{mg} / \mathrm{kg}$ of ketamine (Parnell Laboratories, Auckland, New Zealand) and $11 \mathrm{mg} / \mathrm{kg}$ Xylazine

167 (Rompun, Bayer) delivered in a volume of 0.04-0.05 ml. Additional small doses of

168 anaesthetic were given as required during the course of the experiment. The head was fixed

169 in a custom-made stereotaxic apparatus (Herb Adams, CA) with ear and beak bars and the

170 head was tilted down $28^{\circ}$ from the vertical axis. The head skin was reflected from the

171 midline, the skull surface cleaned and a small metal plate was fixed to the skull using dental

172 cement (Land Dental Manufacturing Co., Inc., Wheeling, IL). This allowed the head to be

173 held at a fixed angle during experimentation after removing the ear bars to present auditory

174 stimulation. The coordinates for electrode insertion were measured with respect to the

175 bifurcation of the mid-sagittal sinus ( $Y$ sinus). A small opening in the skull and dura mater

176 was made over the chosen coordinates to allow electrode penetration into the midbrain.

\section{STIMULUS GENERATION AND PRESENTATION}

178 Auditory stimuli consisted of pure tones, white noise, conspecific and heterospecific

179 songs. Both white noise and tones were generated using Adobe Audition 3.0 software taking PeerJ feviewing PDF | (2019:09:40873:2:0:CHECK 11 May 2020) 
180 into account described hearing ranges for zebra finches (see Okanoya and Dooling 1987).

181 Pure tone stimuli (1, 2, 3, 4, 5 and $6 \mathrm{kHz})$ were of 1s duration with built-in rise and fall ramps

182 of $150 \mathrm{~ms}$ at onset and offset to avoid stimulus clicking through the speaker. The white noise

183 (WN) stimulus was also $1 \mathrm{sec}$ in duration and contained frequencies ranging from 0 to 10

$184 \mathrm{kHz}$. Noise and tone stimuli were presented at $75 \mathrm{~dB}$ SPL, as measured with an SPL meter

185 (RadioShack) $30 \mathrm{~cm}$ from a free-field speaker.

186 The songs used were conspecific (zebra finch) and heterospecific (Bengalese finch)

187 songs. The conspecific songs were chosen from birds raised in the same aviary as the birds

188 used in this experiment and were therefore familiar to both ZF-ZF and ZF-CF birds. They

189 were obtained by recording vocalizations of each individual bird using Sound Analysis Pro

190 software. They were then bandpass filtered $(300 \mathrm{~Hz}-12 \mathrm{kHz})$ using Raven 1.3 software.

191 Heterospecific songs were kindly provided by Dr Sarah Woolley from Columbia University,

192 New York. Song lengths ranged between 1.816s to 2.691s for the conspecific songs and

1931.940 s to 3.091s for the Bengalese finch songs. All songs were calibrated to be presented

194 with an average power of $75 \mathrm{~dB}$ (as calibrated using a $1 / 2$-inch free-field microphone, Brüel

195 and Kjaer and Raven 1.3 software). Songs were played in the forward and backward

196 directions.

197 Each stimulus (white noise, tones and songs) was constructed so as to contain $500 \mathrm{~ms}$

198 of silence preceding and following the actual stimulus. Each stimulus was presented to the

199 bird 15 consecutive times, with different stimuli presented in a randomised order. The

200 interval between the 15 consecutive presentations of a single stimulus type was kept

201 constant within a sequence, but these intervals were randomised for each separate

202 sequence (between 0.5 to $1.5 \mathrm{~s}, 0.1 \mathrm{~s}$ steps). Each unit therefore received 19 stimuli: white

203 noise, 6 pure tones and 12 songs ( 3 conspecific songs in their forward and reverse

204 directions and 3 heterospecific songs in their forward and reverse directions). At the end of

205 the presentation of each set of stimuli, $30 \mathrm{~s}$ of baseline activity for each unit was also

206 recorded at least two minutes after the end of the last stimulus presentation, and this activity

207 was used to calculate the spontaneous firing rate.

208 Stimuli were presented to the bird through a multifunction processor (Tucker-Davis

209 Technology, TDT System 3), routed through an attenuator (TDT) that allowed equal intensity

210 presentation of all stimuli at $75 \mathrm{~dB}$ SPL average power. The signal was then processed

211 through a TDT Stereo Amplifier connected to a free-field magnetic loudspeaker (TDT)

212 placed $30 \mathrm{~cm}$ in front of the bird. Before an experiment, the output of the loudspeaker was

213 checked using a RadioShack SPL meter so to ensure constancy of the average power at

214 which songs were delivered. 


\section{Manuscript to be reviewed}

215

216

217

218

219

220

221

222

223

224

225

226

227

228

229

230

231

232

233

234

235

236

237

238

239

240

241

242

243

244

245

246

247

248

\section{ELECTROPHYSIOLOGICAL RECORDINGS}

All recordings were made in a sound attenuation chamber (Microbooth, All-DUCT Fabrication, PTY. Ltd, Melbourne, Australia), using tungsten or platinum backed electrodes (FHC Inc., Bowdoin, ME; impedances $\sim 10 \mathrm{M} \Omega$ at $1 \mathrm{kHz}$ ). The location of MLd was determined using a variety of search stimuli (white noise, hand claps, clicks, vocalizations), so as to avoid any bias that could result from missing cells responsive to a narrow range of stimuli. Tones were not used as search stimuli, so the data set may contain an underrepresentation of units responsive only to specific tones. The recorded signal was filtered (300 Hz low/5 kHz high pass) and amplified (gain 100x) using an AC amplifier (A-M systems Model 1800) and digitised using the TDT multifunction processor RX6. Threshold and spike discrimination were achieved in real time using the OpenController interface of the TDT OpenEx Suite. Raster plots and peristimulus-time histograms (PSTH) were visualized in real-time using the OpenScope interface, for a rapid qualitative assessment of the auditory selectivity of isolated units prior to the stimulus presentation protocol. The sequence of song presentations and unit recordings were made using a custom-made program designed using the TDT RPvdsEx control (P. Logerot). During the experiment, individual stimuli were chosen from a prebuilt collection of stimuli through the OpenController interface, which also allowed us to set the number of consecutive presentations of the stimulus (15 for this study), the inter-stimulus interval and the attenuation. The order in which stimuli were presented was determined randomly for each series of presentations. The epoch store tracked the onset of each stimulus repeat within a stimulation sequence, and this was then used to align sequential presentations to construct the peristimulus-time histograms and raster plots.

Electrolytic lesions (40 $\mu \mathrm{A}, \mathrm{DC}$ for $10 \mathrm{~s})$ were made to identify and reconstruct recording site locations but, unfortunately, these proved frequently too large to assess the sites with precision. They could, however, be confidently used to confirm the recording site within MLd.

\section{ANALYSIS}

Units were analysed off-line using TDT's OpenSorter and OpenExplorer packages of the OpenEx suite. OpenSorter was used to manually eliminate outlier spikes and to confirm that recordings represented single units. When in doubt, the data were not included in the final analysis. The spontaneous firing rate of the unit (spikes/s) was calculated using the first $20 \mathrm{~s}$ of the $30 \mathrm{~s}$ baseline activity recorded at the end of the session.

PeerJ geviewing PDF | (2019:09:40873:2:0:CHECK 11 May 2020) 
249 Units were classified as 'auditory' when they reached criterion for at least one of the

250 stimuli (whether WN, a tone or a song). Post-stimulus histograms (PSTHs) of $20 \mathrm{~ms}$ bins

251 were constructed using TDT Openexplorer. Neurons were considered to reach the criterion if

252 the responses within at least one $20 \mathrm{msec}$ bin were above threshold, with threshold set at

253 the mean +5 S.D. of the baseline firing rate (defined as the firing rate during the $500 \mathrm{~ms}$ of

254 silence preceding the sound stimulus) (Prather et al. 2009). This choice of threshold biases

255 the population used for analysis, since units that show an inhibition as a result of the

256 auditory stimulus would not be included in the analysis, unless they reached criterion when

257 presented with another stimulus. Once a unit met the criterion for at least one stimulus, the

258 responses to all of the stimuli were considered for the analysis.

RESPONSE STRENGTH

260 Response strength (RS) measures the amount of evoked activity above spontaneous rate. RS is sensitive to changes in levels of evoked activity in response trains that are sustained during the presentation of the stimuli and is also sensitive to inhibitory response. RS was measured for all song stimuli (conspecific, heterospecific, forward and reverse) for all units that met the criteria to be classified as auditory (see above). Response strength was calculated for each song by subtracting the spontaneous activity (in spikes/sec, spontaneous spike rate (SSR)) from the evoked spike rate (spikes/sec, evoked spike rate (ESR)) for each of the 15 consecutive presentations and averaging these values: the spontaneous spike rate and $N$ is the sample size in terms of the number of stimuli. $R S$ values have the disadvantage of not distinguishing changes in the timing of action potentials that may not be accompanied by overall changes in firing rate. Thus, low values of RS could indicate both a lack of response as well as a highly feature-specific response. For example, a unit could show small changes of RS around threshold, yet show a clear change in the temporal pattern of the spikes associated with the song stimulus. 
282

283

284

285

286

287

288

289

290

291

292

293

294

295

296

297

298

299

300

301

302

303

304

305

306

307

308

309

310

311

312

313

314

To compare unit responses between two stimuli we used the Selectivity Index (SI) and d'. Both SI and d' values can be used to assess a unit's selectivity (or 'preference') of one stimulus over another in the pair. Both quantifiers are based on the unit's mean response strength (RS) values for each of the stimuli (and therefore will be influenced by 'inhibited' or time-locked responses). Unlike the comparisons based on the selectivity index, the d' does not provide information regarding the strength of the responses themselves.

SI compares the responses of a neuron to two different stimuli ( $A$ and $B$ ) but does not take into account response variability. The $\mathrm{SI}$ is calculated as:

$$
S I=\frac{R S_{A}}{\mathrm{RS}_{\mathrm{A}}+\mathrm{RS}_{\mathrm{B}}}
$$

Where $S /$ is the Selectivity Index and $R S_{A}$ and $R S_{B}$ are the Response Strength to stimulus $A$ or $B$, respectively. $\mathrm{SI}$ is expressed as values between zero and 1, with SI's close to 1 and zero showing preference for one or the other stimulus and values close to 0.5 indicating that there is no preferential response to either of the two stimuli.

d' measures the difference in response (or preference of a unit) to a pair of stimuli, $A$ and $B$. If the value of d' (of responses to $A$ compared to $B$ ) is greater than zero, it indicates that stimulus $A$ elicited a greater response in the unit than did stimulus B. A d' smaller than zero, indicates that stimulus $B$ elicited a larger response than stimulus $A$, and values equal to zero indicate that the unit responded equally to both stimuli. Differences in the responses to two stimuli (A and B) are calculated by comparing the RS of the unit to each of the two stimuli ( $R S_{A}$ and $R_{B}$, respectively).

The d' is calculated as:

$$
d_{(A \rightarrow B)}^{\prime}=\frac{2\left(\text { mean } \mathrm{RS}_{\mathrm{A}}-\text { mean } \mathrm{RS}_{\mathrm{B}}\right)}{\sqrt{\sigma^{2} \mathrm{RS}_{\mathrm{A}}+\sigma^{2} \mathrm{RS}_{\mathrm{B}}}}
$$

Where $d^{\prime}$ 'is the discriminability/selectivity in response to a pair of stimuli, $A$ and $B, R S_{A}$ and $R S_{B}$ is Response Strength to stimuli $A$ and $B$, respectively, and $\sigma^{2}$ is the variance. One advantage of the d' value is that the differences between the means of the RS for each stimulus is weighted against the variance of their distributions. A second advantage is that the d' is insensitive to sample size.

Which values of SI and d' would be considered as the criterion for selectivity was determined in a way similar to that of Doupe and Solis (1997). For SI, a unit is considered to PeerJ rofiewing PDF | (2019:09:40873:2:0:CHECK 11 May 2020) 
315 be selective when the mean RS of the unit to one stimulus is at least twice that of the other

316 stimulus (i.e., with $\mathrm{SI}=<0.33$ or $\mathrm{SI}>=0.66$ ). For d' a non-selectivity zone interval was chosen

317 in which most neurons showed non-selectivity based on the SI criterion. A criterion of $\left|d^{\prime}\right|=2$

318 was thus set.

\section{GENERALIZED LINEAR MIXED MODEL}

321 We used a Generalized Linear Mixed Model (GLMM) in SAS 9.3 and SPSS 26 for the analysis of the data. This analysis took into account the lack of independence of the individual data points from multiple neurons recorded from different animals, which otherwise may have led to pseudoreplication (for reviews on these issues, see Lazic 2010, Nakagawa and Hauber 2011). The analysis took into account the following: 1) That each isolated single unit in this experiment was presented with 19 different stimulations each repeated 15 times, i.e. the recording session presented 285 stimuli; and 2) that several units were recorded from a single animal.

329 The data were first tested for the normality of the residuals using the Shapiro-Wilk test

330 and then natural log transformed in an attempt to rectify their non-normal distribution.

331 Homogeneity of variance for the residuals was tested using the Levene test and it was confirmed that this assumption was satisfied. The Levene test was 0.13 before transformation and 0.42 after a natural log transformation; therefore, the null hypothesis of homogeneity of variance was retained.

In order to investigate factors influencing the response of units within birds, a GLMM was fitted with bird and unit within bird as random effects to allow for the clustering of units within birds. Bird category was a fixed, between group factor, and song ID, conspecific/heterospecific (CON/HET) and song direction (i.e. song played either forwards or backwards; FOR/REV) were designated as repeated measures using an unstructured covariance matrix model. The GLMM was conducted using a restricted maximal likelihood estimation (REML) procedure and the Kenwood-Rogers approximation for the degrees of freedom (Gurka and Edwards, 2007; McCulloch et al., 2008). The best covariance matrix model (i.e., unstructured) was chosen on the basis of the smallest Akaike Information

344 Criterion (Gurka and Edwards, 2007; McCulloch et al., 2008; Field, 2011). The interactions

345 of bird category, song type, CON/HET and song direction, were initially included as

346 explanatory variables.

347 The general equation (in matrix notation) for the GLMM analysis used was:

$$
y=X \beta+Z u+\varepsilon
$$




\section{Manuscript to be reviewed}

350

in which $y=$ an $\mathrm{N} \times 1$ column vector of outcome variables; $\mathrm{X}=$ an $\mathrm{N} \times p$ matrix of $p$

352 predictor variables (X included Bird Category, Song type (CON/HET), direction (FOR/REV),

353 Song ID and Bird Category $\times$ CON/HET); $\beta=a p \times 1$ column vector of fixed effects

354 regression coefficients; $Z=$ an $N \times q$ matrix of the $q$ random effects related to a fixed $\beta$ ( $Z$

355 included factors Bird ID and unit within bird); $u$ is a $q \times 1$ vector of random effects); and $\varepsilon=$

356 an N x 1 column vector of residuals (Gurka and Edwards, 2007; McCulloch et al., 2008).

357 Initially, the full factorial model was fitted but then non-significant interactions were

358 systematically omitted in search of the best model for the data, as indicated by the lowest

359 AIC (Rouder et al., 2016). This was a model using unstructured covariance, excluding all

360 interactions except for Bird Category $\times$ CON/HET. As with regression, it is appropriate to

361 remove non-significant terms in search of the best model for the data, providing that some

362 independent criterion such as the AIC is used as a guide (Gurka and Edwards, 2007;

363 McCulloch et al., 2008; Rouder et al., 2016).

364 Post-hoc comparisons were conducted using the least square means estimates from the

365 GLMM (Field, 2011). Mann-Whitney U tests were used to compare the distributions and

366 medians of neuronal responses (Field, 2011)

367 In normal reared zebra finches we found that for both sexes there was evidence of a

368 greater response to CON than HET songs (males $P=.001$, females $P=.0003$ ) and that the

369 size of this difference was greater in males than females. Therefore, sex was not introduced

370 in the analysis, since it is unlikely that the uneven distribution of sex between the two groups

371 would influence our results. This is consistent with behavioural data where sex was not

372 found to be a factor in similar rearing experiments (Campbell and Hauber 2009). 


\section{Manuscript to be reviewed}

\section{RESULTS}

375 We sought to describe the basic responses of single units in MLd to simple stimuli (tones and white noise) and to conspecific (zebra finch) and heterospecific (Bengalese finch) song in normal-reared zebra finches, and in zebra finches that were cross-fostered by Bengalese finches. We present the results for those MLd units that reached criterion to be classified as auditory when all stimuli (white noise, tones, and songs, both conspecific and heterospecific) were considered (58 units from normal reared bids, ZF-ZF: 6 birds, 4-14 units/bird; and 20 units in the cross-fostered birds, ZF-CF: 6 birds, 1-6 units/birds, with 1 ZF-ZF unit where responses to HET-3 is missing). In neither control nor cross-fostered groups was there an obvious individual bird effect on the units' response.

\section{SPONTANEOUS ACTIVITY AND RESPONSES TO TONES AND WHITE NOISE}

Most units in the MLd of ZF-ZF and ZF-CF showed no spontaneous activity $(<1$ spike/sec) or showed low spontaneous activity ( $<5$ spikes/sec) (66\% in ZF-ZF, 75\% in ZFCF) (Figure 2). Units with higher spontaneous rates were found in ZF-ZF units, but not in ZFCF midbrain units. As high spontaneous rate units were infrequent in the ZF-ZF birds, our failure to find units with strong discharge rates in cross fostered zebra finches may be related to the smaller number of units recorded.

\section{INSERT FIGURE 2 AROUND HERE}

Unit responses to white noise (WN) and tones are summarised in Table 1. Most ZF-ZF units and all ZF-CF units responded to at least one of the simple stimuli. Unit responses showed mostly excitation, although inhibition was also seen (6 units in ZF-ZF and 3 units in ZF-CF). Most units showed responses to $\mathrm{WN}$ and at least one of the tone stimuli, and most responded to more than one frequency, although 4 units in the ZF-ZF MLd failed to respond to any of the tones.

401

\section{TABLE 1 AROUND HERE}


406 lower frequencies, $2 \mathrm{kHz}$ being the most represented best frequency, followed by 1 and 3

$407 \mathrm{kHz}$ (Table 2).

408

TABLE 2 AROUND HERE

410

The temporal response patterns of each unit are based on a qualitative assessment of the shape of the PSTH obtained in response to BF and to WN and are summarized in Figure $3 \mathrm{E}$. Units were classified as onset, sustained, primary-like or primary-like with notch (Fig. 3A-D). Not all units could be confidently classified into any of these 4 categories based on stimulation with BF or WN. Units showing a sustained response to $\mathrm{BF}$ and $\mathrm{WN}$ were the most frequently found in both ZF-ZF and ZF-CF.

\section{FIGURE 3 AROUND HERE}

Tuning properties and response patterns to tones and WN of MLd units were comparable in the midbrains of ZF-ZF and ZF-CF birds. Thus, our qualitative analysis failed to detect an effect of rearing on the basic tuning properties of MLd neurons to simple stimuli beyond that which may be accounted for by differences in sample size.

\section{EFFECT OF LEARNING IN RESPONSES TO SONG}

426 Behavioural data have shown that normal-reared zebra finches prefer to spatially associate with conspecific song over Bengalese finch song, whereas zebra finches that were cross fostered by Bengalese finches associated equally with both types of song. Using birds from the same population used for these behavioural experiments, we sought to examine the extent to which responses of auditory units in the auditory midbrain mirrored these behavioural differences. We challenged single units in the MLd of normal reared (ZF-ZF) and cross fostered (ZF-CF) zebra finches with 12 song stimuli: 3 conspecific (CON) songs and 3 heterospecific, Bengalese finch (HET) songs. All song stimuli were presented in the forward (FOR) and reverse (REV) direction. Of all the units that were classified as auditory in the present study most (ZF-ZF: 79\%; ZF-CF: 75\%) responded to all types of stimuli (WN, tones, and songs). A minority of neurons responded only to song stimuli (Table 3). Those

437 units where WN failed to elicit a response did respond to both tones and song stimuli. Most

438 units were excited by the stimuli, although inhibition was also seen. One unit in a ZF-CF bird Peer) feyiewing PDF | (2019:09:40873:2:0:CHECK 11 May 2020) 
439 was found to be inhibited by $\mathrm{WN}, 1 \mathrm{kHz}, 2 \mathrm{kHz}$, and $3 \mathrm{kHz}$ stimuli, but excited by $5 \mathrm{kHz}$ and a

440 few song stimuli.

Both conspecific (CON) and heterospecific (HET) song elicited robust responses from MLd units in ZF-ZF and ZF-CF zebra finches. Thirty-four of the 58 units of ZF-ZF (59\%) and 15 of the 20 units of ZF-CF (75\%) responded to all 12 song stimuli. The remaining 24 units $(41 \%)$ did not respond to at least one of the song stimuli (Figure 4$)$. Only $10 \%$ of the ZF-ZF and $15 \%$ of the ZF-CF units responded to 8 songs or less. There was no indication of any bias towards conspecific song or forward version of the song at this level of analysis.

450

\section{FIGURE 4 AROUND HERE}

Response patterns of MLd units of ZF-ZF and ZF-CF birds, were heterogenous, with many units showing strong responses throughout the song stimulus presentation. Qualitatively, responses appeared to be mainly isomorphic, following the AM envelope of the conspecific or heterospecific stimulus (Figure 5A) with fewer units responding in a more sustained manner throughout the entire song stimulus (Fig. 5B). Other unit responses appeared to show some degree of selectivity in their responses, either by responding more selectively to some elements within songs or by responding much more vigorously to some songs in particular (Figure $5 \mathrm{C}$ and $\mathrm{D}$ ).

The type of response to one stimulus was not, however, a good predictor of the unit's response to another one. For example, Figure 6 shows the responses of a ZF-CF MLd unit which is inhibited by white noise (Fig. 6A),, and conspecific song 2 (CON-2) but increases its firing rate in response to heterospecific song 2 (HET-2). (See also Supplementary Table)

468 Note that the increased response at the onset of CON-2 corresponds to a long silent period within the stimulus. Some other units would only respond to a subset of songs. 


\section{FIGURE 6 AROUND HERE}

\section{Manuscript to be reviewed}

472

473 Determining what aspects of the song elicit these different types of responses is beyond 474 the scope of this study. These results underscore the complexity of the neuronal responses 475 encountered in this study, and show that the population of units in this study was not 476 homogeneous with respect to response characteristics.

To compare the responses of units to pairs of stimuli we calculated the selectivity index (SI) and d'. In the present study, a d'A ${ }_{A \rightarrow B}>2$ indicates a neuron's stronger response to stimulus $A$ over stimulus $B$ while a $d_{A \rightarrow B}<-2$ indicates a neuron's stronger response to stimulus $B$ over stimulus $A$. A value of d' falling between [-2, 2] (non-selectivity zone) indicates no preference of one stimulus over the other (see Material and Methods). We plotted the cumulative distribution of the d'values for a given song against its reverse version and the other 5 forward songs for all instances in normal reared birds (58 units $\times 5-1$ comparisons) and for all instances in cross-fostered zebra finches (20 units x 5 comparisons).

Figure 7 shows these distributions of d' for all instances when conspecific song 2 (CON2, in ZF-ZF MLd, Fig. 7A and ZF-CF MLd, Fig 7D) and heterospecific song 1 (HET-1, in ZFZF MLd, Fig. 7C and in ZF-CF MLd, Fig. 7B) were compared against their respective reverse version and all other forward versions of songs. Most comparisons fall within the non-selectivity interval, but many comparisons are found outside of this interval. In the case of $\mathrm{CON}-2$, for those instances showing preference, most prefer $\mathrm{CON}-2$ over its reverse or other forward songs (d' values > 2). As in the ZF-ZF, in cross fostered zebra finches the cumulative distributions of d' values of each song against all others for all instances suggest that, at the population level, there is no preference for an individual song stimulus with most comparisons falling within the non-selectivity zone (Fig. 7B).

\section{FIGURE 7 AROUND HERE}

At the unit level, all but 2 units from ZF-ZF and 1 in ZF-CF MLds showed discrimination to at least one pair of stimuli (d' outside the non-selectivity interval, Fig. 8). A unit's 
503 discrimination between a pair of stimuli could not predict discrimination response to a

504 second pair.

505

FIGURE 8 AROUND HERE

507

Taken together, these results show that the majority of units in the MLd of both ZF-ZF and ZF-CF are able to discriminate at least one feature within the stimulus set.

512 The response strength (RS) measures the amount of activity within the total firing rate

513 that can be directly attributed to the presentation of the stimulus. The RS to each song

514 stimulus of the MLd units recorded in this study ranged from -3.58 to 41.37 spikes/s in ZF-ZF

515 and -0.28 to 17.03 spikes/s in ZF-CF zebra finches, and there was no tail towards high

516 values of RS in ZF-CF zebra finches (Fig. 9). In the ZF-ZF auditory midbrain, other than the

517 peak at 0 , the majority of instances were confined between 0 and 5 spikes/s whereas in ZF-

518 CF higher RS values appear to fall less sharply (Figure 9).

519 Thus, MLd units from cross-fostered zebra finches appeared to have lower RS values

520 and a narrower range of RS values than those in normal reared birds, although formal

521 statistical analysis indicated that the mean difference was not statistically significant $(F 1,15)$

$522=0.33, P=0.58)$. The RS values obtained for the forward and reverse presentation of a

523 given song stimulus in a single unit are plotted against each other in Figure 10 . In $59 \%$ of the

524 instances (here 58 units $\times 6-1$ FOR/REV song comparisons), slightly greater responses of

525 the units toward the reverse version of songs were observed, which was confirmed by

526 GLMM analysis $(F 1,467)=16.87, \mathrm{P}=0.0001)$.

527 In order to investigate factors influencing the response strength of units within birds, a

528 GLMM was fitted with bird and unit within bird as random effects to allow for the clustering of 529 units within birds, and song and direction (i.e. song played either forwards or backwards) as

530 repeated measures with unstructured covariance. Bird Category (reared with conspecifics or

531 cross-tutored), song type (conspecific or heterospecific, CON/HET), song within type

532 (conspecific song 1, 2 or 3; heterospecific song 1, 2 or 3), direction of song (forward or

533 reverse, FOR/REV) as well as the interactions of category, song type and song direction and

534 the interaction of song with direction were initially included as explanatory variables and then 
535 interactions that were not significant were removed from the analysis (Rouder et al., 2016),

536 as explained and justified in the Analysis section of the Methods.

537 Initially, a full factorial model was fitted to the data. Using this model, no significant

538 difference was found between ZF-ZF and ZF-CF birds as a main effect $(F(1,15=0.43, P=$

5390.51 ) but there were significant main effects of individual songs (Song ID) $(F(4,384)=4.4, P$

$540=0.003)$ and the direction of songs (FOR/REV: $F(1,467)=13.26, P=0.0001)$. A reduced

541 model was investigated and compared to the initial model using the AIC values, where the

542 smaller value is better. When the reduced model was used, on the basis of the smaller AIC

543 value (Rouder et al., 2016), the results were, in general, similar: Bird Category as a main

544 effect was non-significant $F(1,15=0.33, P=0.58)$, individual song (Song ID) as a main

545 effect was significant $F(4,384=4.95, P=0.0007)$, as was $F O R / R E V ~ F(1,467=16.87, P=$

546 0.0001). In addition, there was a significant difference between ZF-ZF and ZF-CF birds in

547 the effect of CON or HET, with ZF-ZF birds having larger reductions in response to HET

548 than to CON compared to ZF-CF birds (i.e., a significant interaction: $F(1,384=4.12, P=$

549 0.043; see Tables 4 and 5; Figs. 9B and 10). These appear to result from a reduction in

550 response strength to conspecific song and an increase in response strength to the song of

551 the foster species (mean RS: ZF-ZF= $5.76(\mathrm{CON}), 4.49(\mathrm{HET}) ; \mathrm{ZF}-\mathrm{CF}=5.20(\mathrm{CON}), 5.12$

552 (HET); both $\mathrm{P}=0.0001$; see Table 5$)$. CON/HET was also significant as a main effect but

553 because it was significant in interaction with Bird Category, it was not examined further as

554 an independent effect (Nelder, 1977; Martinez, 2015). Despite this strong evidence that

555 direction influences RS depending on rearing, the size of the effect was not large. This is

556 demonstrated when the RS values obtained for the forward and reverse presentations of a

557 given song stimulus in a single unit are plotted against each other where most data points

558 fall on or near the equality line (Fig. 10).

559 These results show that the MLd units of all birds did not respond equally to individual

560 songs within one category (CON or HET) and indicate that early auditory experience shapes

561 responses in the auditory midbrain in the zebra finch (Table 4).

562 


\section{DISCUSSION}

569 We sought to describe the responses of MLd units in normal reared zebra finches (ZF-

570 ZF) that sing a zebra-finch typical song, and zebra finches that had learned an abnormal 571 song through cross fostering with Bengalese finches (ZF-CF). MLd units in both ZF-ZF and

572 ZF-CF showed comparable responses to white noise and tones. In contrast, birds that were

573 cross-fostered modified the responses of MLd units to conspecific and heterospecific song

574 stimuli in a way that parallels behavioural shifts in song preference in the same population of

575 birds as that used by Campbell and Hauber (2009, 2010). We therefore conclude that the

576 effects of developmental experience, although not limited to hearing and learning non-

577 conspecific vocalizations during development, but which removes the preference for

578 conspecific song in behavioural experiments, are manifested in the response properties of

579 brainstem auditory neurons.

580 GENERAL DESCRIPTION

SPONTANEOUS RATE

582 In agreement with previous studies (Woolley and Casseday 2004) most of our MLd units 583 showed no or low spontaneous activity. Woolley and Casseday (2004) reported 90\% of their 584 units had no spontaneous activity, with an upper limit of 7 spikes/s. In contrast, we found 585 that units with no spontaneous activity were less common (22\% in ZF-ZF and $20 \%$ in ZF-

$586 \mathrm{CF}$ ), and some units in ZF-ZF MLd had spontaneous rates to $14-15$ spikes/s. These 587 discrepancies are not surprising given that Woolley and Casseday used urethane as an 588 anaesthetic in contrast to ketamine/xylazine in this study. When compared to 589 unanaesthetized birds, Schumacher et al. (2011) showed that MLd units in urethane 590 anaesthetized male zebra finches had lower spontaneous activity than in unanaesthetized 591 birds. Similarly, in the inferior colliculus of the guinea pig, Astl et al. (1996) found lower rates 592 of spontaneous activity under urethane anaesthesia than under ketamine anaesthesia.

593 Thus, discrepancies in the range of spontaneous activity are likely attributable to the 594 anaesthetic regime. We did observe, however, that MLd units from ZF-CF finches showed a 595 narrower range of spontaneous activity than those from ZF-ZF finches. Woolley et al. (2010) 596 do not report if cross-fostering results in changes in spontaneous activity, although they do 597 report that cross-fostering reduces the firing rate of MLd units. Since in normal-reared zebra 598 finches units with high spontaneous activity are infrequent, it is possible that these 599 differences are the result of a smaller sample size.

600 
601

602

603

604

605

606

607

608

609

610

611

612

613

614

615

616

617

618

619

620

621

622

623

624

625

626

627

628

629

630

631

632

633

634

635

636

RESPONSE CHARACTERISTICS

Most of the units we recorded from responded to all types of stimuli (WN, tones, song: $79 \%$ of ZF-ZF units and $75 \%$ of ZF-CF units, Table 3 ), the remainder responding to a subset of the stimuli. Consistent with previous work, we found several temporal response patterns in MLd units from ZF-ZF and ZF-CF finches when stimulated with best frequency (BF) and white noise (WN) (Woolley and Casseday 2004, Schumacher et al. 2011). Fewer units could be classified into a given temporal response pattern when stimulated with WN than with $B F$, but the proportion of units that could be classified into any one temporal response pattern was similar in ZF-ZF and ZF-CF. The most frequent temporal response pattern in both bird groups was the sustained type, followed by the onset type. The literature reports varying proportions of temporal response patterns. Schumacher et al. (2011) found the primary-like response to be the most frequently found in the MLds of unanaesthetized and urethane anaesthetized zebra finches. In contrast, Woolley and Casseday (2004) found in urethane anaesthetized zebra finches that onset responses were the most frequent. The discrepancies between these two studies suggest that the differences in proportions of response types may be due to sampling biases between different studies. For example, in the inferior colliculus (IC) of the cat, the onset pattern was reported as the most common type by Rose et al. (1963), whereas Aitkin et al. (1994) report that the sustained units predominate over onset units. Thus, while providing descriptive value of the population at hand, differences in the proportion of response patterns may be difficult to interpret meaningfully.

Zebra finch and Bengalese finch songs have a comparable frequency range and both have harmonic stacks in their song, but diverge in other spectro-temporal elements. Zebra finch songs usually contain noisy and harmonic elements whereas Bengalese songs contain fast repeated harmonic syllables called trills (Zann 1996, Woolley and Casseday 2004). Zebra finches cross-fostered with Bengalese finch parents learn a song that contains BF elements within temporal gaps typical of zebra finch songs (Araki et al. 2016). Both zebra finch (CON) and Bengalese finch (HET) songs elicited robust isomorphic and nonisomorphic responses, but the response to one stimulus was not necessarily a good predictor of the unit's response to another. For example, frequency tuning alone could not explain the selective response to complex vocalizations. This mirrors results obtained in guinea fowl (Scheich et al. 1977), zebra finches (Woolley et al. 2006, Schneider and Woolley 2011), bats (Portfors 2004), and fish (Maruska and Tricas 2009). Although our study was not designed to identify the precise features that evoked the unit's response, some degree of feature extraction in MLd was evident. Single units showed discrimination between the varied song types, although not all songs were preferred equally by MLd units, and no single PeerJ 20 riewing PDF | (2019:09:40873:2:0:CHECK 11 May 2020) 
637 song was consistently preferred. Some units responded to all songs and all elements within

638 the songs, others selectively responded to subsets of songs or to particular spectro-temporal

639 features within notes or syllables, whereas others were more tightly tuned to the amplitude

640 modulations. Woolley and Casseday (2005) report that $79 \%$ of the units they recorded from

641 in the male zebra finch MLd responded to amplitude modulated tones. Woolley et al. (2009)

642 using spectral-temporal receptive fields (STRFs) to analyse the responses of zebra finch

643 midbrain neurons to conspecific songs, identified four functional groups, each with the

644 potential to contribute to feature extraction of the conspecific signals. More than half were

645 broadband neurons whose characteristic STRF made them either good at detecting onsets

646 and suitable for encoding rhythm, or good at encoding amplitude envelope and suitable to

647 participate in encoding of timbre. Distinct populations dedicated to encoding either spectral

648 features or temporal information have also been described in fish (for review see Bass and

649 McKibben 2003) amphibians (for review, see Feng et al. 1990, Bass et al. 2005, Rose and

650 Gooler 2007), and mammals (for review see Ehret and Schreiner 2005, Rees and Langner

$6512005)$. In the present study we were able to detect subpopulations within MLd that could

652 potentially contribute to the discrimination of conspecific vocalisations, providing further

653 evidence that there is some degree of feature extraction at the level of MLd. Furthermore,

654 the d' and SI data suggest that cross fostering does not influence the ability of units to

655 discriminate between natural stimuli. The heterogeneity of responses to conspecific stimuli in

656 the auditory midbrain, together with a key role of inhibition in shaping the selectivity of

657 neurons, has been described; lifting inhibition leads to a broader tuning curve and a

658 loosening of the neuron's selectivity to complex vocalizations (Yang et al. 1992, Hall 1999,

659 Klug et al. 2002, Portfors 2004, Xie et al. 2005, Pollak 2011, Mayko et al. 2012). In zebra

660 finches Woolley and Portfors (2013) showed that blocking GABA and glycine inhibition

661 results in an expansion of the tuning curves of IC neurons and reduces the selectivity to

662 vocalizations. It will be interesting to determine whether the reduced discrimination between

663 CON-HET categories that is seen as a result of cross-fostering is associated with changes in

664 GABAergic function in the MLd.

665

667 We sought to answer two questions: Do units in MLd show a response bias towards 668 conspecific songs, and if this exists, can this be modified by developmental experience? To 669 this end, we challenged MLd units of ZF-ZF and ZF-CF zebra finches with stimuli consisting 670 of 3 familiar zebra finch (CON) and 3 unfamiliar Bengalese finch (HET) songs, each played 671 forwards and backwards. We found that in both groups of birds, responses to song stimuli 
672 were heterogeneous, that units in MLd were able to discriminate between different stimuli,

673 and that a preference towards conspecific song was developmentally modified.

674 The response parameter of choice for the GLMM analysis was RS because it better

675 reflects the responses of the units to different song stimuli. While the $\mathrm{SI}$ is good at detecting

676 selectivity to certain features, its shortcoming lies in its failure to detect the strength of that

677 response. The RS, although it provides little information regarding the temporal pattern of

678 the evoked response, is a more direct measure of the 'total' response of the cell. The

679 distribution of RS values in ZF-CF differs from that seen with ZF-ZF units (Mann-Whitney U

680 test for equality of distributions, $P=0.001$ ), although the mean differences were non-

681 significant. Woolley et al. (Woolley et al. 2010) showed that cross-fostering resulted in a

682 lower firing rate in MLd neurons. In contrast we find that the median RS for cross fostered

683 birds is higher than for normal-reared birds (ZF-ZF: 2.4, ZF-CF: 4.4) (Mann-Whitney U test

684 for medians, $P=0.0001$ ). Schumacher et al. (2011) found that units in MLd of male zebra

685 finches under urethane anaesthesia had lower spontaneous and song-evoked firing rates

686 and response strength (compared to unanaesthetised birds). In guinea pig IC, Astl et al.

687 (1996) showed that frequency tuning was similar under urethane versus Ketamine/Xylazine

688 anaesthesia, although the number of spontaneously active neurons was higher under

689 Ketamine/Xylazine. While the anaesthetic regime may account for differences in overall

690 response levels between our and Woolley's results, it is unlikely to account for the direction

691 of the changes observed as a result of cross-fostering.

692 Three main findings resulted from the GLMM analysis of RS values. First, the reverse

693 versions of songs evoked stronger responses than the forward versions of songs in both ZF-

694 ZF and ZF-CF zebra finches. Second, there was an effect of individual songs within CON

695 and HET categories. Third, ZF-ZF MLd units responded more strongly to CON than to HET

696 songs than did their ZF-CF counterparts. Each of these findings will be considered

697 separately below.

698

The stronger responses toward reverse songs is puzzling. Woolley and Casseday (2005) found that in MLd, $89 \%$ of the units they tested were responsive to FM sweeps, but most of these cells were insensitive to the direction of the sweep. Of the five cells that showed a preference, four preferred the upward sweep direction. Since the zebra finch song contains more downward FM than upward FM, a preference for upward sweeps would be expected to evoke a stronger response to reverse song in FM-sensitive neurons. The ICs of bats are 
707 (Suga 1965, Fuzessery and Hall 1996, Casseday et al. 1997, Razak and Fuzessery 2006,

708 Andoni et al. 2007, Voytenko and Galazyuk 2007) and express a range of preferences for

709 sweep velocities that correspond to the sweep velocities in the signals they emit (Andoni et

710 al. 2007). However, and in contrast to our neural data, Lauay et al (2004) found no

711 significant difference in a choice task in the preference for forward song over reverse song in

712 zebra finch females, whether they had been reared with both parents or isolated from male

713 tutors. A preference for forward and reverse version of song in cross-fostered zebra finches

714 has, to our knowledge not been examined.

717 Our results show that the MLd units of both ZF-ZF and ZF-CF birds did not respond

718 equally to individual songs within one category (CON or HET). At the population level, not all

719 songs were preferred equally, and no single song was consistently preferred. However,

720 single-units were able to discriminate between the varied song types. The heterogeneity of

721 responses in MLd appears to include a large population of neurons that represent a large

722 range of stimulus characteristics, with units showing highly reproducible responses that

723 indicate a high degree of selectivity to stimulus parameters. More interestingly, the analysis

724 also showed that there was an effect of song within either the conspecific or heterospecific

725 signals. Thus, the choice of stimuli used for these kinds of studies, and whether they are

726 considered an independent variable, might have an impact on the interpretation of the

727 results.

EFFECT OF REARING IN THE PREFERENCE TO CONSPECIFIC SONG

730 Selectivity toward the forward conspecific over the heterospecific songs in the MLd of

731 ZF-ZF and ZF-CF birds was tested by Woolley et al. (2010). Contrary to the results

732 presented here, they did not find a difference in the responses to conspecific and

733 heterospecific songs in male ZF-ZF or ZF-CF, although the mean firing rates to both types of

734 stimuli were lower in the cross-fostered birds. In contrast, our study shows that response

735 differences between CON/HET categories is reduced as a result of rearing experience, likely

736 due to a reduction in response strength to conspecific song and an increase in response

737 strength to the song of the foster species. Differences in the cross-fostering paradigm, the

738 choice of songs as stimuli, or effects of the use of different anaesthetics may account for the

739 discrepancies in the two studies. However, our neural data mirrors the behavioural

740 preference reported in the same population of birds (Campbell and Hauber 2009, 2010).

PeerJ f3riewing PDF | (2019:09:40873:2:0:CHECK 11 May 2020) 
741 More likely, the discrepancies may result from the regions of MLd that were sampled.

742 Although Woolley et al. (2010) do not report on the location of their recordings, the BF of the

743 units in our study suggest our recordings were confined to more dorsal aspects of MLd

744 (Scheich et al. 1977, Coles and Aitkin 1979, Calford et al. 1985, Takahashi and Konishi

745 1988, Woolley and Casseday 2004). In the guinea fowl Scheich et al. (1977) report that

746 recording from ventral MLd yielded units whose responses to calls were predictable from the

747 unit's response to tones, whereas recordings from posterior-dorsal areas yielded a higher

748 proportion of units whose response to complex calls could not be predicted from the

749 neuron's tuning curve. Regional differences in the response to complex stimuli have also

750 been reported in the IC of cat (Aitkin et al. 1994) and guinea pigs (Lyzwa et al. 2016) and in

751 the torus of frogs (Hoke et al. 2004, Mangiamele and Burmeister 2011).

752 The importance of rearing environment in the development of preference for 753 communication sounds in birds is well known. Swamp sparrows, for example, show a

754 behavioural preference for conspecific over heterospecific signals (Dooling and Searcy 755 1980). Adult male and female zebra finches will prefer conspecific over heterospecific songs when challenged with a behavioural choice task, regardless of whether they have been

757 raised in isolation from a male tutor or with both conspecific parents (Braaten and Reynolds 758 1999, Lauay et al. 2004). Juvenile male and female zebra finches that were reared in 759 acoustic isolation preferred conspecific song over heterospecific song (Braaten and

760 Reynolds 1999). Females raised with an adult male preferred the songs of tutored males

761 over those of untutored males, while females that were deprived of the presence of a male

762 did not show this preference (Lauay et al. 2004). Normally-reared birds, exposed solely to 763 conspecifc vocalizations, respond preferentially to conspecific songs. But when deprived of 764 the conspecific exposure and instead develop hearing a Bengalese finch song, zebra 765 finches present similar responses to the songs of both their own species and those of the 766 cross-fostering species.

We did not establish whether the responses we observed are influenced by ascending or descending inputs to MLd (Pannese et al. 2015). In mammals, subcortical structures have been shown to contribute to the processing of vocalizations (Bauer et al. 2002, Pannese et al. 2015, Roberts and Portfors 2015), but recordings from the lower brainstem in songbirds have been limited to studies of tonotopy (Konishi 1969, Konishi 1970, Sachs and Sinnott 1978). To our knowledge, no attempts have been made to examine the responses of

775 hindbrain auditory nuclei in the processing of complex sounds to infer what influence they 
776 may have on MLd responses. It should be noted, however, that the terminal fields of

777 ascending inputs to MLd originating in the hindbrain do not conform to the typical avian

778 pattern where the nucleus laminaris (NL) terminal field defines an MLd core (Conlee and

779 Parks 1986, Takahashi and Konishi 1988). Instead, the terminal fields appear to overlap

780 more broadly within MLd (Krützfeldt et al. 2010). The organization of the NL of songbirds

781 also shows morphological modifications, the significance of which remains unknown (Kubke

782 and Carr 2006). Whether these anatomical differences reflect an adaptation for specific

783 aspects of sound processing has not been established.

784 Descending inputs to the auditory midbrain have been shown to influence neuronal

785 responses in a number of vertebrates (Endepols and Walkowiak 1999, Gao and Suga 2000,

786 Suga et al. 2000, Endepols and Walkowiak 2001, Ma and Suga 2001, Suga et al. 2002,

787 Popelár et al. 2016). It is thus possible that some of the changes observed in MLd may be

788 influenced by forebrain auditory nuclei (Gentner and Margoliash 2003, Cousillas et al. 2004, 789 Jeanne et al. 2011, George and Cousillas 2013, Araki et al. 2016). The auditory forebrain

790 has been implicated in the categorization of vocalizations (Chew et al. 1996, Grace et al.

791 2003, Elie and Theunissen 2015). Strong responses to conspecific song have been

792 recorded in Field $\mathrm{L}$, and the development of neuronal selectivity for conspecific song

793 appears to parallel the emergence of behavioural song preference (Amin et al. 2007).

794 Changes in forebrain responses that are influenced by development have also been

795 reported in the ventral caudal hyperstriatum (cHV) (Grace et al. 2003) and caudal medial

796 nidopallium (Stripling et al. 2001, Menardy et al. 2012). However, descending inputs to MLd

797 in songbirds are not substantial. The only descending input that has been described is from

798 the robust nucleus of the arcopallium (RA) cup (Mello et al. 1998), but these inputs are

799 largely confined to a region external to MLd, and the adjacent intercollicular complex.

800 Similarly with respect to the RA cup's projection to the vicinity of lower auditory brainstem

801 nuclei, such as the dorsal and ventral nuclei of the lateral lemniscus (LLD and LLV), both of 802 which project strongly to MLd (Wild et al. 2010). But in neither the case of MLd nor in that of

803 LLD and LLV have injections of neural tracers been found to retrogradely label adjacent

804 neurons in the region of the RA cup's descending projections.

805

806

\section{CONCLUSION}

807 One interesting observation in our study is that the differences in responses to conspecific and heterospecific songs between ZF-ZF and ZF-CF finches were present even

809 though both groups of birds were housed in the same aviary as adults, thus exposed to the

810 same sound environment. This suggests that the differences in response to conspecific and PeerJ 25iewing PDF | (2019:09:40873:2:0:CHECK 11 May 2020) 
811 heterospecific songs recorded in MLd are not simply a result of repeated exposure to a

812 particular auditory stimulus. Repeated exposure has been shown to change neural

813 responses in the auditory cortex of rats (Bao et al. 2013), ferrets (Schnupp et al. 2006), and

814 gerbils (Caras and Sanes 2017), and in the auditory midbrain of mice (Cruces-Solís et al.

815 2018) and frogs (Gall and Wilczynski 2014). Instead, the persistence of the differences

816 observed in the MLd of both groups of birds, suggest that these changes may be influenced

817 by the critical period of learning associated with song learning. A 'memory' of learned song

818 in the MLd of zebra finches has been suggested by van der Kant et al. (2013).

819 Our results further confirm that neurons in MLd are tuned to characteristics of the

820 conspecific song that allow discrimination between conspecific and heterospecific learned

821 vocalisations. These neuronal 'filters' can be modified during the period of song learning in

822 the direction that would support the learning of a heterospecific song. Further studies in

823 juvenile birds will be needed to determine the developmental trajectory of these changes,

824 and how that trajectory maps onto changes in ascending and descending inputs and the

825 emergence of behavioural preference for conspecific song.

826

827 
828 Figure legends

829 Figure 1: The avian song system and the avian ascending auditory system. (A)

830 Schematic showing the premotor pathway involved in vocal output (nuclei and connections

831 labelled in green) and the Anterior Forebrain Pathway involved in song learning (nuclei and

832 connections labelled in orange). (B) Schematic showing the ascending connections of the

833 auditory pathway. Grey arrows indicate a parallel pathway for auditory input into the song

834 system, Abbreviations: HVC (proper name), RA (robustus archopallialis), DM (dorsal medial

835 nucleus of the intercollicular complex), nXIlts (trachosyringeal portion of the hypoglossal

836 nucleus), RAm/Pam (nuclei retroambigualis/parambigualis), Uva (n. uvaeformis), NIf (n.

837 interface), MMAN (medial portion of the magnocellular $n$. of the anterior nidopallium), LMAN

838 (lateral portion of the magnocellular $n$. of the anterior nidopallium), AreaX (proper name),

839 DMP (dorsomedial nucleus of the posterior thalamus), DLM (medial portion of the

840 dorsolateral anterior thalamic nucleus), Bas (basorostral $\mathrm{n}$. of the pallium), OB (olfactory

841 bulb), CM (caudo- medial mesopallium), NCM (caudal medial nidopallium), L1 (proper

842 name), L2 (proper name), L3 (proper name), Ov (ovoid n. of the thalamus), MLd (n.

843 Mesencephalicus lateralis pars dorsalis, ie. auditory torus), LLD (dorsal $n$. of the lateral

844 lemniscus), LLI (intermediate n. of the lateral lemniscus), LLV (ventral n. of the lateral

845 lemniscus), OS (superior olive), NL (n. laminaris), CN (cochlear nuclei, i.e., n.

846 magnocellularis and $\mathrm{n}$. angularis).

847 Figure 2: Distribution of spontaneous rate of the units recorded in MLd. Most units show 848 spontaneous rates below 1 spike/sec. Black bars: ZF-ZF, gray bars: ZF-CF. Note difference 849 in $Y$ axis scale.

$850 \quad$ Figure 3: Examples of the four temporal response patterns found in the normal zebra

851 finch MLd. All PSTHs in this figure have $20 \mathrm{~ms}$ bins on the time axis and represent response

852 of units to their best frequency. The pure tone lasted $1 \mathrm{~s}$ and was preceded and followed by

$853500 \mathrm{~ms}$ of silence. (A) PSTH of a unit classified as onset showing with a typical strong

854 response at the onset of the stimulus (yellow line in the underlying spectrogram) followed by

855 either no or little response during the rest of the stimulation. (B) PSTH of a unit classified as

856 sustained showing continued evoked response throughout the stimulus presentation. (C)

857 PSTH of a unit classified as primary-like showing a strong response at the onset of the

858 stimulus, followed by a continued but less vigorous firing rate during the duration of the rest

859 of the stimulus. (D) PSTH of a unit classified as primary-like with notch, with a response

860 pattern similar to that of the primary-like response but with a visible dip in firing rate shortly

861 after stimulus onset. (E) Table showing the percentages (number) of units per temporal 
862 response patterns to their best frequency (BF) and to white noise (WN) stimulation in ZF-ZF 863 and ZF-CF birds.

864 Figure 4: Distribution of units of ZF-ZF (black) and ZF-CF (gray) responding to different

865

866

867

868

869

870

871

872

873

874

875

876

877

878

879

880

881

882

883

884

885

886

887

888

889

890

891

892

893

894

895 number of songs. In both normal reared (ZF-ZF) and cross-fostered (ZF-CF) zebra finches, most units responded to all 12 song stimuli, and in both groups, units were found to respond to a small number of the song stimuli.

Figure 5: Examples of response patterns to song in MLd units from normal reared zebra finches (ZF-ZF). The black arrows indicate the beginning of the sound stimulus and of the unit's response. (A) Example of a unit where the responses are locked to the AM envelope of the song and responded to most, if not all the components of the song. (B) Example of a unit responding with a sustained pattern throughout the presentation of the song. ( $C$ and $D)$ Examples of the same unit's response to a conspecific and heterospecific song. In response to conspecific song $(C)$ the unit shows a strong responses to two but not all harmonic stacks (grey arrows and white arrows respectively). In response to a heterospecific song that does not contain harmonic stacks, the same unit shows strong responses to the first series of trills, but not to the second half of the trills (white arrows).

Figure 6. Inhibition and excitation by different stimuli in the same MLd unit of a crossfostered zebra finch (ZF-CF). The black arrows indicate the beginning of the sound stimulus and of the unit's response. This unit showed inhibition in response to white noise $(A)$ and a conspecific song $(B)$, but is excited with the heterospecific song.

Figure 7: Cumulative distribution of $d$ ' values. (A) Cumulative distribution of $d$ ' values for the 58 individual MLd units recorded from in response to conspecific song 2 (CON-2) against its reverse version and all other forward song stimuli in normal reared zebra finches (ZF-ZF). (B) Cumulative distribution of d' values for the 20 individual MLd units recorded from for heterospecific song 1 (HET-1) against its reverse version and all other forward song stimuli in cross-fostered zebra finches (ZF-CF). (C) Cumulative distribution of d' values for the 58 individual MLd units recorded from in response to heterospecific song 1 (HET-1) against its reverse version and all other forward song stimuli in normal reared zebra finches (ZF-ZF). (D) Cumulative distribution of d' values for the 20 individual MLd units recorded from for conspecific song 2 (CON-2) against its reverse version and all other forward song stimuli in cross-fostered zebra finches (ZF-CF). In all plots black-filled circles represent non-selective instances as determined by SI value and unfilled circles represent selective instances as determined by SI value (see Methods) The vertical black line delimit the non-selectivity zone where d'>|2|. 
896 Figure 8 Distribution of d' values. (A) Distribution of d' values for 58 MLd units from

897 normal reared zebra finches (ZF-ZF) for conspecific song 2 (CON-2) against other forward

898 song stimuli.(B) Distribution of d'values for $20 \mathrm{MLd}$ units from cross-fostered zebra finches

899 (ZF-CF) for conspecific song 2 (CON-2) against all other forward song stimuli. (C)

900 Distribution of d' values for 58 MLd units from normal reared zebra finches (ZF-ZF) for

901 heterospecific song 1 (HET-1) against other forward song stimuli. (D) Distribution of d'

902 values for $20 \mathrm{MLd}$ units from cross-fostered zebra finches (ZF-CF) for heterospecific song 1

903 (HET-1) against all other forward song stimuli. (In all plots the horizontal black lines delimit

904 the non-selectivity zone where d'>|2|. Data points above the non-selectivity zone indicate

905 instances in which CON-2 (A and B) or HET-1 (C and D) were preferred over the other

906 songs they were tested against. Data points below the non-selectivity zone indicate

907 instances in which the other song stimuli were preferred over CON-2 (A and B) or HET-1 (C 908 and D).

909 Figure 9 Distribution of RS values. (A) Distribution of RS values (spikes/sec) for all song

910 stimuli presentations from MLd units from normal reared (ZF-ZF, black bars) and cross-

911 fostered (ZF-CF, grey bars) zebra finches. (B) RS (spikes/sec) values for ZF-ZF (black) and

912 ZF-CF (gray) for all units in response to conspecific (CON) or heterospecific (HET) stimuli.

913 The range and mean of the responses are indicated for each group.

914 Figure 10 Mean RS value of individual units. Plots of mean RS values for each individual

915 unit in response to a forward song vs. its reverse version. (A) normal reared zebra finches

916 (ZF-ZF); (B) cross-fostered zebra finches (ZF-CF). In both plots the diagonal line indicates

917 equal response to forward and reverse version. (C) and (D) Histograms showing the

918 minimum distance of individual data points to the identity line calculated as: (RS to FOR

919 version of the song + RS to REV version of the song $/ \sqrt{ } 2$. Positive values were assigned to

920 instances where response to FOR song is larger than to REV song and negative values

921 were assigned to instances where responses to REV song are larger than to FOR song.

922 Percentages indicate the proportion of units that fall either above, or below the identity line.

923 
924 Acknowledgements

925

926

927

928

929

930

931

932

933

934

935

936

937

938

939

940

941

942

943

944

945

946

947

948

949

950

951

952

953

954

955

956

957

958

959

960

961

962

963

964

965

966

967

968

969

970

This work was funded by the Marsden Fund, and previously published as a PhD thesis (Logerot, 2011). We would like to thank Dana Cambpell for providing the birds used in this study, and Joanna Stewart for initial assistance with statistical analysis.

\section{References}

Aitkin, L., L. Tran and J. Syka (1994). "The responses of neurons in subdivisions of the inferior colliculus of cats to tonal, noise and vocal stimuli." Experimental Brain Research 98(1): 53-64.

Amin, N., A. Doupe and F. E. Theunissen (2007). "Development of Selectivity for Natural Sounds in the Songbird Auditory Forebrain." Journal of Neurophysiology 97(5): 3517-3531.

Andoni, S., N. Li and G. D. Pollak (2007). "Spectrotemporal Receptive Fields in the Inferior Colliculus Revealing Selectivity for Spectral Motion in Conspecific Vocalizations." Journal of Neuroscience 27(18): 4882-4893.

Araki, M., M. M. Bandi and Y. Yazaki-Sugiyama (2016). "Mind the gap: Neural coding of species identity in birdsong prosody." Science 354(6317): 1282-1287.

Astl, J., J. Popelář, E. Kvašňák and J. Syka (1996). "Comparison of Response Properties of Neurons in the Inferior Colliculus of Guinea Pigs Under Different Anesthetics." Audiology 35(6): 335-345.

Bao, S., E. F. Chang, C. L. Teng, M. A. Heiser and M. M. Merzenich (2013). "Emergent categorical representation of natural, complex sounds resulting from the early postnatal sound environment." Neuroscience 248: 30-42.

Bass, A. H. and J. R. McKibben (2003). "Neural mechanisms and behaviors for acoustic communication in teleost fish." Progress in Neurobiology 69(1): 1-26.

Bass, A. H., G. J. Rose and M. B. Pritz (2005). Auditory Midbrain of Fish, Amphibians, and Reptiles: Model Systems for Understanding Auditory Function. The Inferior Colliculus. J. A. Winer and C. E. Schreiner, Springer New York: 459-492.

Bauer, E. E., A. Klug and G. D. Pollak (2002). "Spectral Determination of Responses to Species-Specific Calls in the Dorsal Nucleus of the Lateral Lemniscus." Journal of Neurophysiology 88(4): 1955-1967.

Bodnar, D. A., A. D. Holub, B. R. Land, J. Skovira and A. H. Bass (2001). "Temporal population code of concurrent vocal signals in the auditory midbrain." Journal of Comparative Physiology A 187(11): 865-873.

Bolhuis, J. J. and S. Moorman (2015). "Birdsong memory and the brain: In search of the template." Neuroscience \& Biobehavioral Reviews 50: 41-55.

Braaten, R. F. and K. Reynolds (1999). "Auditory preference for conspecific song in isolation-reared zebra finches." Animal Behaviour 58(1): 105-111.

Brainard, M. S. and A. J. Doupe (2000). "Auditory feedback in learning and maintenance of vocal behaviour." Nature Reviews Neuroscience 1(1): 31-40.

Calford, M. B., L. Z. Wise and J. D. Pettigrew (1985). "Coding of sound location and frequency in the auditory midbrain of diurnal birds of prey, families accipitridae and falconidae." Journal of Comparative Physiology A 157: 149-160.

Campbell, D. L. M. and M. E. Hauber (2009). "Cross-fostering diminishes song discrimination in zebra finches (Taeniopygia guttata)." Animal Cognition 12(3): 481490.

Campbell, D. L. M. and M. E. Hauber (2010) "Conspecific-only experience during development reduces the strength of heterospecific song discrimination in Zebra 


\section{Manuscript to be reviewed}

971

972

973

974

975

976

977

978

979

980

981

982

983

984

985

986

987

988

989

990

991

992

993

994

995

996

997

998

999

1000

1001

1002

1003

1004

1005

1006

1007

1008

1009

1010

1011

1012

1013

1014

1015

1016

1017

1018

1019

1020

1021

1022

1023
Finches (Taeniopygia guttata): a test of the optimal acceptance threshold hpothesis." Journal of Ornithology 151:379-389.

Caras, M. L. and D. H. Sanes (2017). "Top-down modulation of sensory cortex gates perceptual learning." Proceedings of the National Academy of Sciences 114(37): 9972-9977.

Casseday, J. H., E. Covey and B. Grothe (1997). "Neural selectivity and tuning for sinusoidal frequency modulations in the inferior colliculus of the big brown bat, Eptesicus fuscus." J Neurophysiol 77: 1595-1605.

Catchpole, C. K. and P. J. B. Slater (2003). Bird Song: Biological Themes and Variations, Cambridge University Press.

Chew, S. J., D. S. Vicario and F. Nottebohm (1996). "A large-capacity memory system that recognizes the calls and songs of individual birds." Proceedings of the National Academy of Sciences 93(5): 1950-1955.

Clayton, N. S. (1989). "The Effects of Cross-Fostering on Selective Song Learning in Estrildid Finches." Behaviour 109(3/4): 163-175.

Coles, R. B. and L. M. Aitkin (1979). "The response properties of auditory neurones in the midbrain of the domestic fowl (Callus gallus) to monaural and binaural stimuli." Journal of comparative physiology 134(3): 241-251.

Conlee, J. W. and T. N. Parks (1986). "Origin of ascending auditory projections to the nucleus mesencephalicus lateralis pars dorsalis in the chicken." Brain Research 367(1): 96-113.

Cousillas, H., J.-P. Richard, M. Mathelier, L. Henry, I. George and M. Hausberger (2004). "Experience-dependent neuronal specialization and functional organization in the central auditory area of a songbird: Specialization in songbird cenatal auditory area." European Journal of Neuroscience 19(12): 3343-3352.

Covey, E. and C. E. Carr (2005). The Auditory Midbrain in Bats and Birds. The Inferior Colliculus. J. A. Winer and C. E. Schreiner. New York, NY, Springer New York: 493536.

Cruces-Solís, H., Z. Jing, O. Babaev, J. Rubin, B. Gür, D. Krueger-Burg, N. Strenzke and L. d. Hoz (2018). "Auditory midbrain coding of statistical learning that results from discontinuous sensory stimulation." PLOS Biology 16(7): e2005114.

Dooling, R. and M. Searcy (1980). "Early perceptual selectivity in the swamp sparrow." Developmental Psychobiology 13(5): 499-506.

Doupe, A. J. and M. M. Solis (1997). "Song- and order-selective neurons develop in the songbird anterior forebrain during vocal learning." Journal of Neurobiology 33(5): 694-709.

Eales, L. A. (1987). "Do zebra finch males that have been raised by another species still tend to select a conspecific song tutor?" Animal Behaviour 35(5): 1347-1355.

Ehret, G. and C. E. Schreiner (2005). Spectral and Intensity Coding in the Auditory Midbrain. The Inferior Colliculus. J. A. Winer and C. E. Schreiner. New York, NY, Springer New York: 312-345.

Elie, J. E. and F. E. Theunissen (2015). "Meaning in the avian auditory cortex: neural representation of communication calls." European Journal of Neuroscience 41(5): 546-567.

Endepols, H. and W. Walkowiak (1999). "Influence of descending forebrain projections on processing of acoustic signals and audiomotor integration in the anuran midbrain." European Journal of Morphology 37(2-3): 182-184.

Endepols, H. and W. Walkowiak (2001). "Integration of ascending and descending inputs in the auditory midbrain of anurans." Journal of Comparative Physiology A 186(12): 1119-1133.

Feng, A. S., J. C. Hall and D. M. Gooler (1990). "Neural basis of sound pattern recognition in anurans." Progress in Neurobiology 34(4): 313-329.

Field, A. (2011) "Discovering Statistics Using SPSS (3rd Ed.)”, Sage, California. 
1024 Fuzessery, Z. M. and J. C. Hall (1996). "Role of GABA in shaping frequency tuning and

1025

1026

1027

1028

1029

1030

1031

1032

1033

1034

1035

1036

1037

1038

1039

1040

1041

1042

1043

1044

1045

1046

1047

1048

1049

1050

1051

1052

1053

1054

1055

1056

1057

1058

1059

1060

1061

1062

1063

1064

1065

1066

1067

1068

1069

1070

1071

1072

1073

1074

1075

1076

1077 creating FM sweep selectivity in the inferior colliculus." Journal of Neurophysiology 76(2): 1059-1073.

Gall, M. D. and W. Wilczynski (2014). "Prior experience with conspecific signals enhances auditory midbrain responsiveness to conspecific vocalizations." The Journal of Experimental Biology 217(11): 1977-1982.

Gao, E. and N. Suga (2000). "Experience-dependent plasticity in the auditory cortex and the inferior colliculus of bats: Role of the corticofugal system." Proceedings of the National Academy of Sciences 97(14): 8081-8086.

Gentner, T. Q. and D. Margoliash (2003). "Neuronal populations and single cells representing learned auditory objects." Nature 424(6949): 669.

George, I. and H. Cousillas (2013). "How social experience shapes song representation in the brain of starlings." Journal of Physiology-Paris 107(3): 170-177.

Grace, J. A., N. Amin, N. C. Singh and F. E. Theunissen (2003). "Selectivity for Conspecific Song in the Zebra Finch Auditory Forebrain." Journal of Neurophysiology 89(1): 472-487.

Gurka, M.J., Edwards, L.J. (2007) "Mixed models". In Handbook of Statistics: Essential Statistical Methods for Medical Statistics. (Eds) C.R. Rao, J.P. Miller, Rao, D.C., Elsevier, NY, pp. 146-173.

Hall, J. C. (1999). "GABAergic inhibition shapes frequency tuning and modifies response properties in the auditory midbrain of the leopard frog." Journal of Comparative Physiology A 185(5): 479-491.

Hoke, K. L., S. S. Burmeister, R. D. Fernald, A. S. Rand, M. J. Ryan and W. Wilczynski (2004). "Functional Mapping of the Auditory Midbrain during Mate Call Reception." Journal of Neuroscience 24(50): 11264-11272.

Holmstrom, L. A., L. B. M. Eeuwes, P. D. Roberts and C. V. Portfors (2010). "Efficient Encoding of Vocalizations in the Auditory Midbrain." Journal of Neuroscience 30(3): 802-819.

Jeanne, J. M., J. V. Thompson, T. O. Sharpee and T. Q. Gentner (2011). "Emergence of Learned Categorical Representations within an Auditory Forebrain Circuit." Journal of Neuroscience 31(7): 2595-2606.

Kennedy, M. C. (1974). "Auditory Multiple-Unit Activity in the Midbrain of the Tokay Gecko (Gekko gecko, L.)." Brain, Behavior and Evolution 10(1-3): 257-264.

Klug, A., E. E. Bauer, J. T. Hanson, L. Hurley, J. Meitzen and G. D. Pollak (2002). "Response selectivity for species-specific calls in the inferior colliculus of Mexican free-tailed bats is generated by inhibition." J Neurophysiol 88: 1941-1954.

Knudsen, D. P. and T. Q. Gentner (2010). "Mechanisms of song perception in oscine birds." Brain and Language 115(1): 59-68.

Konishi, M. (1969). "Hearing, Single-Unit Analysis, and Vocalizations in Songbirds." Science 166(3909): 1178-1181.

Konishi, M. (1970). "Comparative neurophysiological studies of hearing and vocalizations in songbirds." Zeitschrift für vergleichende Physiologie 66(3): 257-272.

Krützfeldt, N. O. E., P. Logerot, M. F. Kubke and J. M. Wild (2010). "Connections of the auditory brainstem in a Songbird, Taeniopygia guttata. I. Projections of nucleus angularis and nucleus laminaris to the auditory torus." Journal of Comparative Neurology 518(11): 2109-2134.

Kubke, M. F. and C. E. Carr (2006). "Morphological Variation in the Nucleus Laminaris of Birds." International Journal of Comparative Psychology 19(1): 83-97.

Kubke, M. F. and J. M. Wild (2018). Anatomy of Vocal Communication and Hearing in Rodents. Rodent Bioacoustics. M. L. Dent, R. R. Fay and A. N. Popper. Cham, Springer International Publishing: 131-164.

Lauay, C., N. M. Gerlach, E. Adkins-Regan and T. J. DeVoogd (2004). "Female zebra finches require early song exposure to prefer high-quality song as adults." Animal Behaviour 68(6): 1249-1255. 
1078 Lazic, S. E. (2010). "The problem of pseudoreplication in neuroscientific studies: is it

1079

1080

1081

1082

1083

1084

1085

1086

1087

1088

1089

1090

1091

1092

1093

1094

1095

1096

1097

1098

1099

1100

1101

1102

1103

1104

1105

1106

1107

1108

1109

1110

1111

1112

1113

1114

1115

1116

1117

1118

1119

1120

1121

1122

1123

1124

1125

1126

1127

1128

1129

1130 affecting your analysis?" BMC Neuroscience 11(1): 5.

Logerot, P. (2011). Anatomical organisation, auditory processing of communication signals and role of auditory experience in the auditory midbrain of the zebra finch. PhD $\mathrm{PhD}$, University of Auckland.

Lyzwa, D., J. M. Herrmann and F. Wörgötter (2016). "Natural Vocalizations in the Mammalian Inferior Colliculus are Broadly Encoded by a Small Number of Independent Multi-Units." Frontiers in Neural Circuits 9: 91.

$\mathrm{Ma}, \mathrm{X}$. and N. Suga (2001). "Corticofugal modulation of duration-tuned neurons in the midbrain auditory nucleus in bats." Proceedings of the National Academy of Sciences 98(24): 14060-14065.

Mangiamele, L. A. and S. S. Burmeister (2011). "Auditory selectivity for acoustic features that confer species recognition in the túngara frog." Journal of Experimental Biology 214(17): 2911-2918.

Martinez, H.D.R. (2015) "Analyzing interactions with fitted models". CRAN.R. PROJECT. https://cran.r-project.org/web/packages/phia/vignettes/phia.pdf

Maruska, K. P. and T. C. Tricas (2009). "Encoding properties of auditory neurons in the brain of a soniferous damselfish: response to simple tones and complex conspecific signals." Journal of Comparative Physiology A 195(11): 1071-1088.

Mayko, Z., P. D. Roberts and C. Portfors (2012). "Inhibition shapes selectivity to vocalizations in the inferior colliculus of awake mice." Frontiers in Neural Circuits 6 : 73.

McCulloch, C.E., Searle, S.R., Neuhaus, J.M. (2008) “Generalized, Linear and Mixed Models ( $\left.2^{\text {nd }} E d.\right)$." Wiley, NJ.

Mello, C. V., E. Vates, S. Okuhata and F. Nottebohm (1998). "Descending auditory pathways in the adult male zebra finch (Taeniopygia Guttata)." Journal of Comparative Neurology 395(2): 137-160.

Menardy, F., K. Touiki, G. Dutrieux, B. Bozon, C. Vignal, N. Mathevon and C. D. Negro (2012). "Social experience affects neuronal responses to male calls in adult female zebra finches." European Journal of Neuroscience 35(8): 1322-1336.

Nakagawa, S. and M. E. Hauber (2011). "Great challenges with few subjects: Statistical strategies for neuroscientists." Neuroscience \& Biobehavioral Reviews 35(3): 462473.

Nelder, J.A. (1977) "A reformulation of linear models". Journal of the Royal Statistical Society. Series A (General). 140 (1): 48-77.

Nottebohm, F., J. A. Paton and D. B. Kelley (1982). "Connections of vocal control nuclei in the canary telencephalon." The Journal of Comparative Neurology 207(4): 344-357.

Nottebohm, F., T. M. Stokes and C. M. Leonard (1976). "Central control of song in the canary, Serinus canarius." The Journal of Comparative Neurology 165(4): 457-486.

Okanoya, K. and R. J. Dooling (1987). "Hearing in passerine and psittacine birds: A comparative study of absolute and masked auditory thresholds." Journal of Comparative Psychology 101(1): 7-15.

Pannese, A., D. Grandjean and S. Frühholz (2015). "Subcortical processing in auditory communication." Hearing Research 328: 67-77.

Pollak, G. D. (2011). "Discriminating among complex signals: the roles of inhibition for creating response selectivities." Journal of Comparative Physiology A 197(5): 625640.

Pollak, G. D. (2013). "The dominant role of inhibition in creating response selectivities for communication calls in the brainstem auditory system." Hearing Research 305: 86101.

Popeláŕ, J., D. Šuta, J. Lindovský, Z. Bureš, K. Pysanenko, T. Chumak and J. Syka (2016). "Cooling of the auditory cortex modifies neuronal activity in the inferior colliculus in rats." Hearing Research 332: 7-16.

Peer] ऊ̧ฬiewing PDF | (2019:09:40873:2:0:CHECK 11 May 2020) 
1131 Portfors, C. V. (2004). "Combination sensitivity and processing of communication calls in the

1132

1133

1134

1135

1136

1137

1138

1139

1140

1141

1142

1143

1144

1145

1146

1147

1148

1149

1150

1151

1152

1153

1154

1155

1156

1157

1158

1159

1160

1161

1162

1163

1164

1165

1166

1167

1168

1169

1170

1171

1172

1173

1174

1175

1176

1177

1178

1179

1180

1181

1182

1183

inferior colliculus of the Moustached Bat Pteronotus parnellii." Anais da Academia

Brasileira de Ciências 76(2): 253-257.

Portfors, C. V. and D. G. Sinex (2005). Coding of Communication Sounds in the Inferior Colliculus. The Inferior Colliculus. J. A. Winer and C. E. Schreiner. New York, NY, Springer New York: 411-425.

Prather, J. F., S. Nowicki, R. C. Anderson, S. Peters and R. Mooney (2009). "Neural correlates of categorical perception in learned vocal communication." Nature Neuroscience 12(2): 221-228.

Razak, K. A. and Z. M. Fuzessery (2006). "Neural Mechanisms Underlying Selectivity for the Rate and Direction of Frequency-Modulated Sweeps in the Auditory Cortex of the Pallid Bat." Journal of Neurophysiology 96(3): 1303-1319.

Rees, A. and G. Langner (2005). Temporal Coding in the Auditory Midbrain. The Inferior Colliculus. J. A. Winer and C. E. Schreiner. New York, NY, Springer New York: 346376.

Roberts, P. D. and C. V. Portfors (2015). "Responses to Social Vocalizations in the Dorsal Cochlear Nucleus of Mice." Frontiers in Systems Neuroscience 9: 172.

Rodríguez, F. A., H. L. Read and M. A. Escabí (2009). "Spectral and Temporal Modulation Tradeoff in the Inferior Colliculus." Journal of Neurophysiology 103(2): 887-903.

Rose, G. J. and D. M. Gooler (2007). Function of the amphibian central auditory system. Hearing and Sound communication in Amphibians. P. M. Narins, A. S. Feng and R. R. Fay, Berlin, Germany: Springer. 28: 250-290.

Rose, G. J., C. J. Leary and C. J. Edwards (2011). "Interval-counting neurons in the anuran auditory midbrain: factors underlying diversity of interval tuning." Journal of Comparative Physiology A 197(1): 97-108.

Rose, J. E., D. D. Greenwood, J. M. Goldberg and J. E. Hind (1963). "Some discharge characteristics of single neurons in the inferior colliculus of the cat. i. tonotopical organization, relation of spike-counts to tone intensity, and firing patterns of single elements." Journal of Neurophysiology 26(2): 294-320.

Rouder, J.N., Engelhardt, C.R., McCabe, S., Morey, R.D. (2016) "Model comparison in ANOVA". Psychon. Bull. Rev. 23: 1779-1786.

Sachs, M. B. and J. M. Sinnott (1978). "Responses to tones of single cells in nucleus magnocellularis and nucleus angularis of the redwing blackbird (<Emphasis Type="Italic" $>$ Agelaius phoeniceus $</$ Emphasis $>$ )." Journal of Comparative Physiology 126(4): 347-361.

Sayegh, R., B. Aubie and P. A. Faure (2011). "Duration tuning in the auditory midbrain of echolocating and non-echolocating vertebrates." Journal of Comparative Physiology A 197(5): 571-583.

Scheich, H., G. Langner and R. Koch (1977). "Coding of narrow-band and wide-band vocalizations in the auditory midbrain nucleus (MLD) of the Guinea Fowl (Numida meleagris)." Journal of Comparative Physiology 117(2): 245-265.

Schneider, D. M. and S. M. N. Woolley (2010). "Discrimination of Communication Vocalizations by Single Neurons and Groups of Neurons in the Auditory Midbrain." Journal of Neurophysiology 103(6): 3248-3265.

Schneider, D. M. and S. M. N. Woolley (2011). "Extra-Classical Tuning Predicts StimulusDependent Receptive Fields in Auditory Neurons." Journal of Neuroscience 31(33): 11867-11878.

Schnupp, J. W. H., T. M. Hall, R. F. Kokelaar and B. Ahmed (2006). "Plasticity of Temporal Pattern Codes for Vocalization Stimuli in Primary Auditory Cortex." Journal of Neuroscience 26(18): 4785-4795.

Schumacher, J. W., D. M. Schneider and S. M. N. Woolley (2011). "Anesthetic state modulates excitability but not spectral tuning or neural discrimination in single auditory midbrain neurons." Journal of Neurophysiology 106(2): 500-514.

Peer) ऊeyiewing PDF | (2019:09:40873:2:0:CHECK 11 May 2020) 
1184 Stripling, R., A. A. Kruse and D. F. Clayton (2001). "Development of song responses in the

1185

1186

1187

1188

1189

1190

1191

1192

1193

1194

1195

1196

1197

1198

1199

1200

1201

1202

1203

1204

1205

1206

1207

1208

1209

1210

1211

1212

1213

1214

1215

1216

1217

1218

1219

1220

1221

1222

1223

1224

1225

1226

1227

1228

1229

1230

1231

1232

1233

1234

1235

1236

1237 zebra finch caudomedial neostriatum: Role of genomic and electrophysiological activities." Journal of Neurobiology 48(3): 163-180.

Suga, N. (1965). "Analysis of frequency-modulated sounds by auditory neurones of echolocating bats." The Journal of Physiology 179(1): 26-53.

Suga, N., E. Gao, Y. Zhang, X. Ma and J. F. Olsen (2000). "The corticofugal system for hearing: Recent progress." Proceedings of the National Academy of Sciences 97(22): 11807-11814.

Suga, N., Z. Xiao, X. Ma and W. Ji (2002). "Plasticity and Corticofugal Modulation for Hearing in Adult Animals." Neuron 36(1): 9-18.

Šuta, D., E. Kvašňák, J. Popelář and J. Syka (2003). "Representation of Species-Specific Vocalizations in the Inferior Colliculus of the Guinea Pig." Journal of Neurophysiology 90(6): 3794-3808.

Takahashi, T. T. and M. Konishi (1988). "Projections of the cochlear nuclei and nucleus laminaris to the inferior colliculus of the barn owl." Journal of Comparative Neurology 274(2): 190-211.

Takahasi, M., H. Kagawa, M. Ikebuchi and K. Okanoya (2006). "Case studies of song and call learning by a hybrid Bengalese-Zebra Finch and Bengalese-fostered Zebra Finches: Assessing innate factors in vocal learning." Ornithological Science 5(1): 85-93.

Van der Kant, A., S. Derégnaucourt, M. Gahr, A. V. d. Linden and C. Poirier (2013). "Representation of Early Sensory Experience in the Adult Auditory Midbrain: Implications for Vocal Learning." PLOS ONE 8(4): e61764.

Vonderschen, K. and M. J. Chacron (2011). "Sparse and dense coding of natural stimuli by distinct midbrain neuron subpopulations in weakly electric fish." Journal of Neurophysiology 106(6): 3102-3118.

Voytenko, S. V. and A. V. Galazyuk (2007). "Intracellular Recording Reveals Temporal Integration in Inferior Colliculus Neurons of Awake Bats." Journal of Neurophysiology 97(2): 1368-1378.

Wenstrup, J. J., K. Nataraj and J. T. Sanchez (2012). "Mechanisms of spectral and temporal integration in the mustached bat inferior colliculus." Frontiers in Neural Circuits 6: 75.

Wilczynski, W. and M. J. Ryan (2010). "The behavioral neuroscience of anuran social signal processing." Current Opinion in Neurobiology 20(6): 754-763.

Wild, J. M., N. O. E. Krützfeldt and M. F. Kubke (2010). "Connections of the auditory brainstem in a songbird, Taeniopygia guttata. III. Projections of the superior olive and lateral lemniscal nuclei." The Journal of Comparative Neurology 518(11): 21492167.

Woolley, S. M. N. (2012). "Early experience shapes vocal neural coding and perception in songbirds." Developmental Psychobiology 54(6): 612-631.

Woolley, S. M. N. and J. H. Casseday (2004). "Response Properties of Single Neurons in the Zebra Finch Auditory Midbrain: Response Patterns, Frequency Coding, Intensity Coding, and Spike Latencies." Journal of Neurophysiology 91(1): 136-151.

Woolley, S. M. N. and J. H. Casseday (2005). "Processing of Modulated Sounds in the Zebra Finch Auditory Midbrain: Responses to Noise, Frequency Sweeps, and Sinusoidal Amplitude Modulations." Journal of Neurophysiology 94(2): 1143-1157.

Woolley, S. M. N., T. E. Fremouw, A. Hsu and F. E. Theunissen (2005). "Tuning for spectrotemporal modulations as a mechanism for auditory discrimination of natural sounds." Nature Neuroscience 8(10): 1371-1379.

Woolley, S. M. N., P. R. Gill, T. Fremouw and F. E. Theunissen (2009). "Functional Groups in the Avian Auditory System." Journal of Neuroscience 29(9): 2780-2793.

Woolley, S. M. N., P. R. Gill and F. E. Theunissen (2006). "Stimulus-Dependent Auditory Tuning Results in Synchronous Population Coding of Vocalizations in the Songbird Midbrain." Journal of Neuroscience 26(9): 2499-2512. 
1238

1239

1240

1241

1242

1243

1244

1245

1246

1247

1248

1249

1250

1251

1252

1253

1254
Woolley, S. M. N., M. E. Hauber and F. E. Theunissen (2010). "Developmental experience alters information coding in auditory midbrain and forebrain neurons." Developmental Neurobiology 70(4): 235-252.

Woolley, S. M. N. and C. V. Portfors (2013). "Conserved mechanisms of vocalization coding in mammalian and songbird auditory midbrain." Hearing Research 305: 45-56.

Xie, R., J. Meitzen and G. D. Pollak (2005). "Differing Roles of Inhibition in Hierarchical Processing of Species-Specific Calls in Auditory Brainstem Nuclei." Journal of Neurophysiology 94(6): 4019-4037.

Yang, L., G. D. Pollak and C. Resler (1992). "GABAergic circuits sharpen tuning curves and modify response properties in the mustache bat inferior colliculus." Journal of Neurophysiology 68(5): 1760-1774.

Zann, R. (1990). "Song and call learning in wild zebra finches in south-east Australia." Animal Behaviour 40(5): 811-828.

Zann, R. A. (1996). The zebra finch: a synthesis of field and laboratory studies. Oxford; New York, Oxford University Press. 


\section{Figure 1}

The avian song system and the avian ascending auditory system

(A) Schematic showing the premotor pathway involved in vocal output (nuclei and connections labelled in green) and the Anterior Forebrain Pathway involved in song learning (nuclei and connections labelled in orange). (B) Schematic showing the ascending connections of the auditory pathway. Grey arrows indicate a parallel pathway for auditory input into the song system, Abbreviations: HVC (proper name), RA (robustus archopallialis), DM (dorsal medial nucleus of the intercollicular complex), nXIIts (trachosyringeal portion of the hypoglossal nucleus), RAm/Pam (nuclei retroambigualis/parambigualis), Uva (n. uvaeformis), NIf ( $n$. interface), MMAN (medial portion of the magnocellular $n$. of the anterior nidopallium), LMAN (lateral portion of the magnocellular $\mathrm{n}$. of the anterior nidopallium), AreaX (proper name), DMP (dorsomedial nucleus of the posterior thalamus), DLM (medial portion of the dorsolateral anterior thalamic nucleus), Bas (basorostral n. of the pallium), OB (olfactory bulb), CM (caudo- medial mesopallium) , NCM (caudal medial nidopallium), L1 (proper name), L2 (proper name), L3 (proper name), Ov (ovoid $n$. of the thalamus), MLd ( $\mathrm{n}$. Mesencephalicus lateralis pars dorsalis, ie. auditory torus), LLD (dorsal $n$. of the lateral lemniscus), LLI (intermediate $\mathrm{n}$. of the lateral lemniscus), LLV (ventral $\mathrm{n}$. of the lateral lemniscus), OS (superior olive), NL (n. laminaris), CN (cochlear nuclei, i.e., n. magnocellularis and $n$. angularis). 


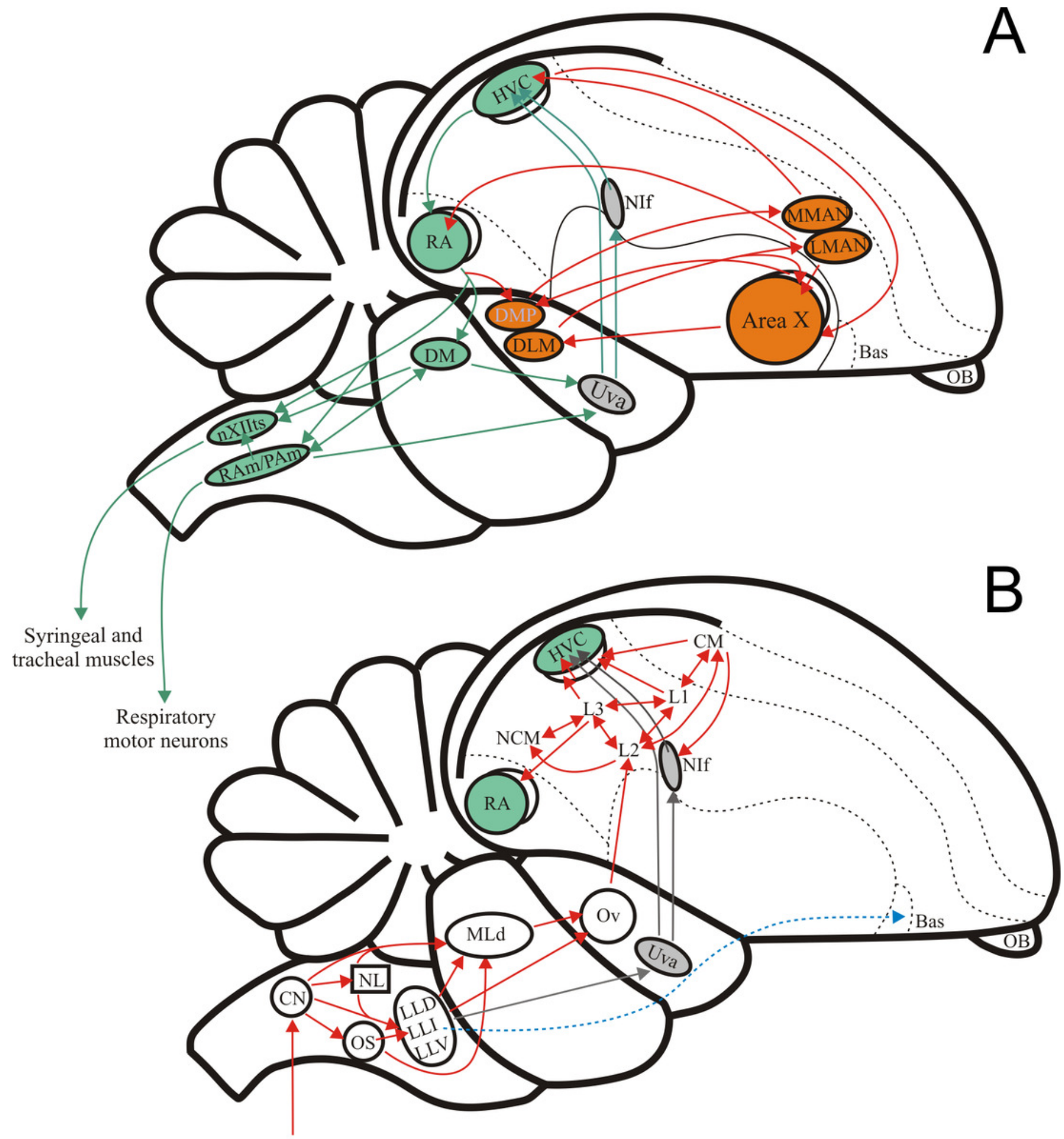

Auditory nerve 
Figure 2

Distribution of spontaneous rate of the units recorded in MLd

Most units show spontaneous rates below 1 spike/sec. Black bars: ZF-ZF, gray bars: ZF-CF. Note difference in $\mathrm{Y}$ axis scale.

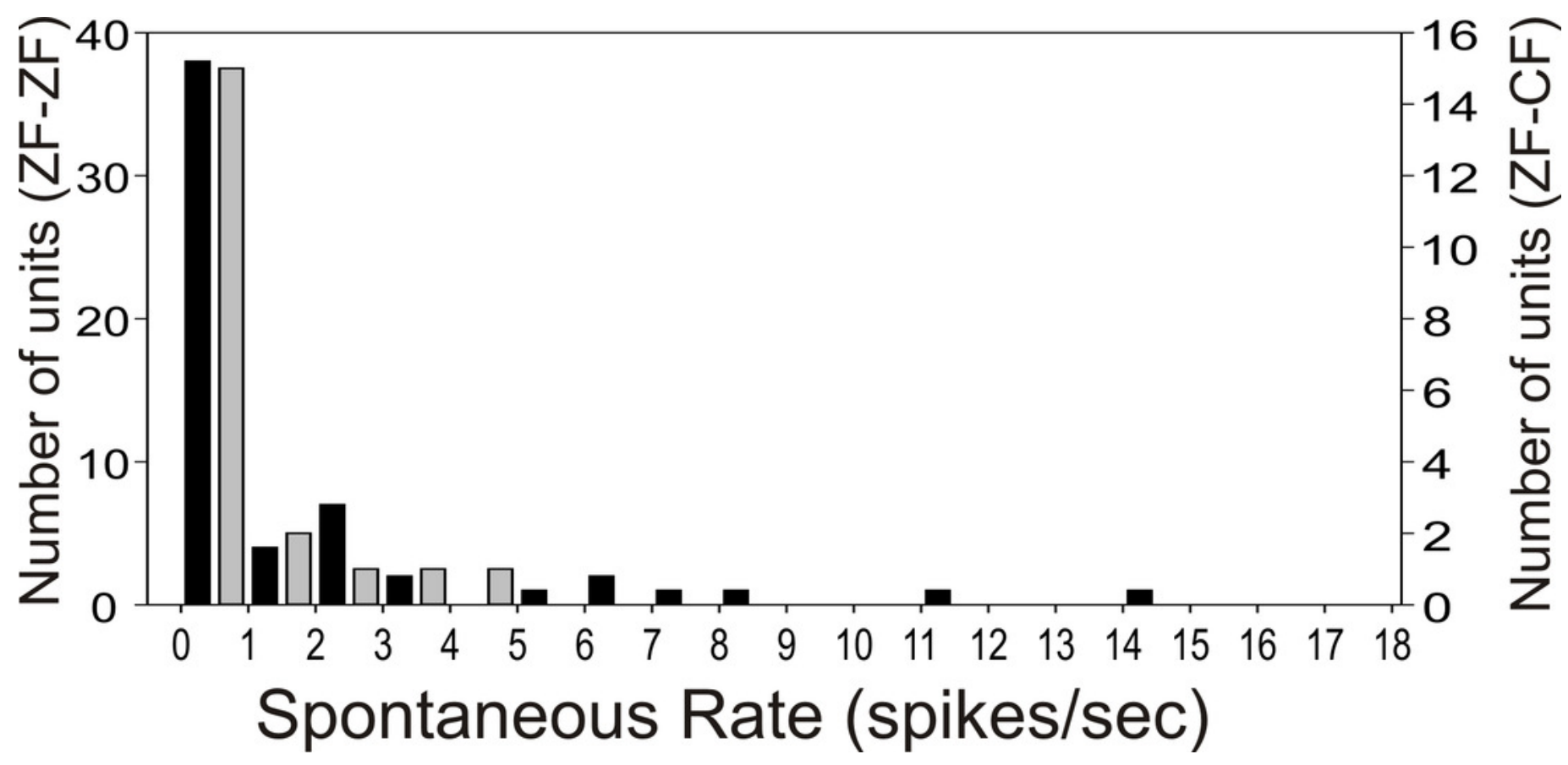




\section{Figure 3}

Examples of the four temporal response patterns found in the normal zebra finch MLd All PSTHs in this figure have $20 \mathrm{~ms}$ bins on the time axis and represent response of units to their best frequency. The pure tone lasted $1 \mathrm{~s}$ and was preceded and followed by $500 \mathrm{~ms}$ of silence. (A) PSTH of a unit classified as onset showing with a typical strong response at the onset of the stimulus (yellow line in the underlying spectrogram) followed by either no or little response during the rest of the stimulation. (B) PSTH of a unit classified as sustained showing continued evoked response throughout the stimulus presentation. (C) PSTH of a unit classified as primary-like showing a strong response at the onset of the stimulus, followed by a continued but less vigorous firing rate during the duration of the rest of the stimulus. (D) PSTH of a unit classified as primary-like with notch, with a response pattern similar to that of the primary-like response but with a visible dip in firing rate shortly after stimulus onset. (E) Table showing the percentages (number) of units per temporal response patterns to their best frequency (BF) and to white noise (WN) stimulation in ZF-ZF and ZF-CF birds. 

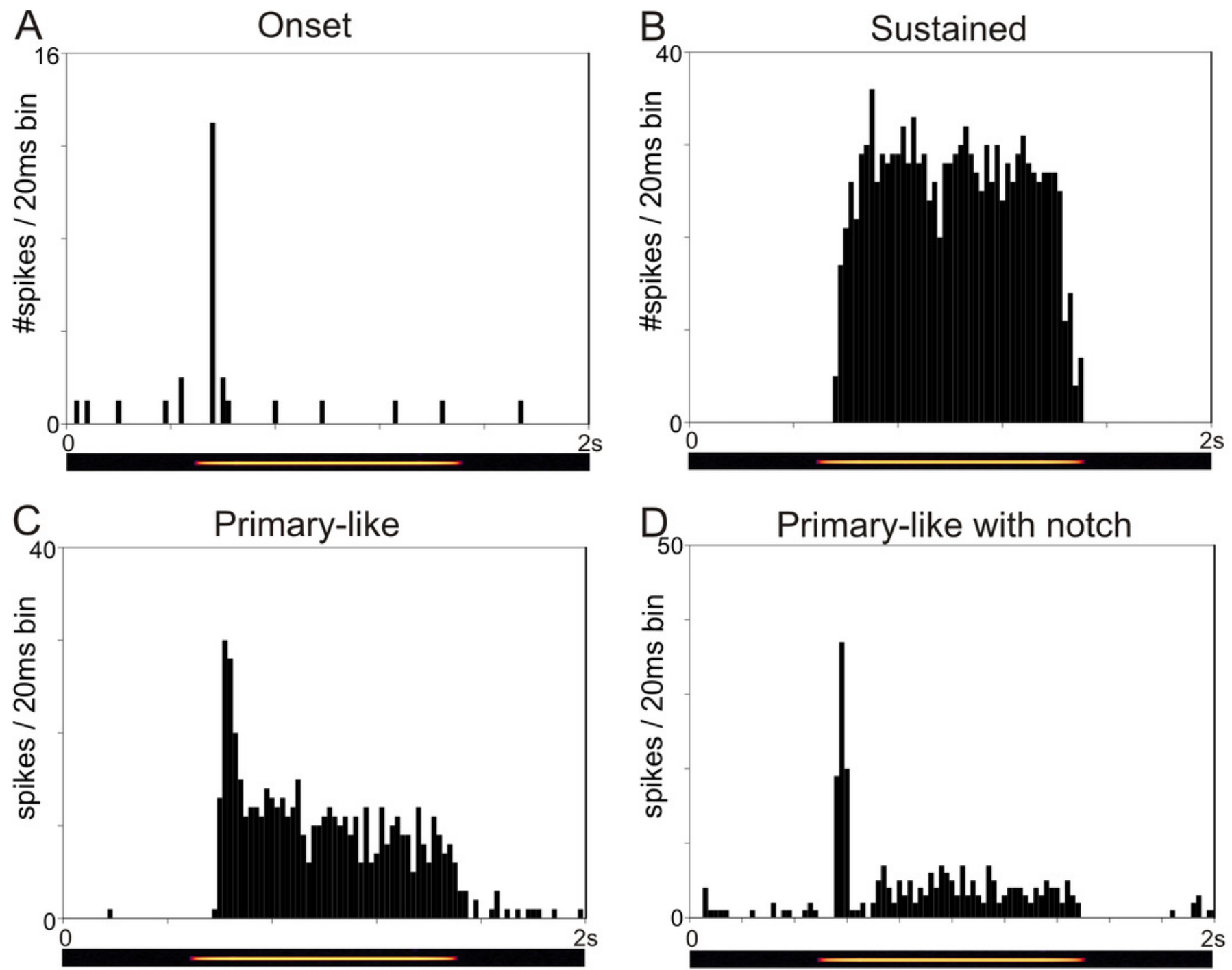

E

\begin{tabular}{ccccccc} 
Stimulus & Onset & Sustained & Primary-like & $\begin{array}{c}\text { Primary-like } \\
\text { with notch }\end{array}$ & $\begin{array}{c}\text { Could not } \\
\text { classify }\end{array}$ \\
\hline \multirow{2}{*}{$\mathrm{BF}$} & ZF-ZF & $14 \%(8)$ & $41 \%(24)$ & $7 \%(4)$ & $7 \%(4)$ & $31 \%(18)$ \\
& ZF-CF & $15 \%(3)$ & $30 \%(6)$ & $5 \%(1)$ & $15 \%(3)$ & $35 \%(7)$ \\
\hline \multirow{2}{*}{ WN } & ZF-ZF & $7 \%(4)$ & $22 \%(13)$ & $5 \%(3)$ & $3 \%(2)$ & $62 \%(36)$ \\
& ZF-CF & $5 \%(1)$ & $30 \%(6)$ & $0 \%(0)$ & $5 \%(1)$ & $60 \%(12)$ \\
\hline
\end{tabular}


Figure 4

Distribution of units of ZF-ZF (black) and ZF-CF (gray) responding to different number of songs.

In both normal reared (ZF-ZF) and cross-fostered (ZF-CF) zebra finches, most units responded to all 12 song stimuli, and in both groups, units were found to respond to a small number of the song stimuli.

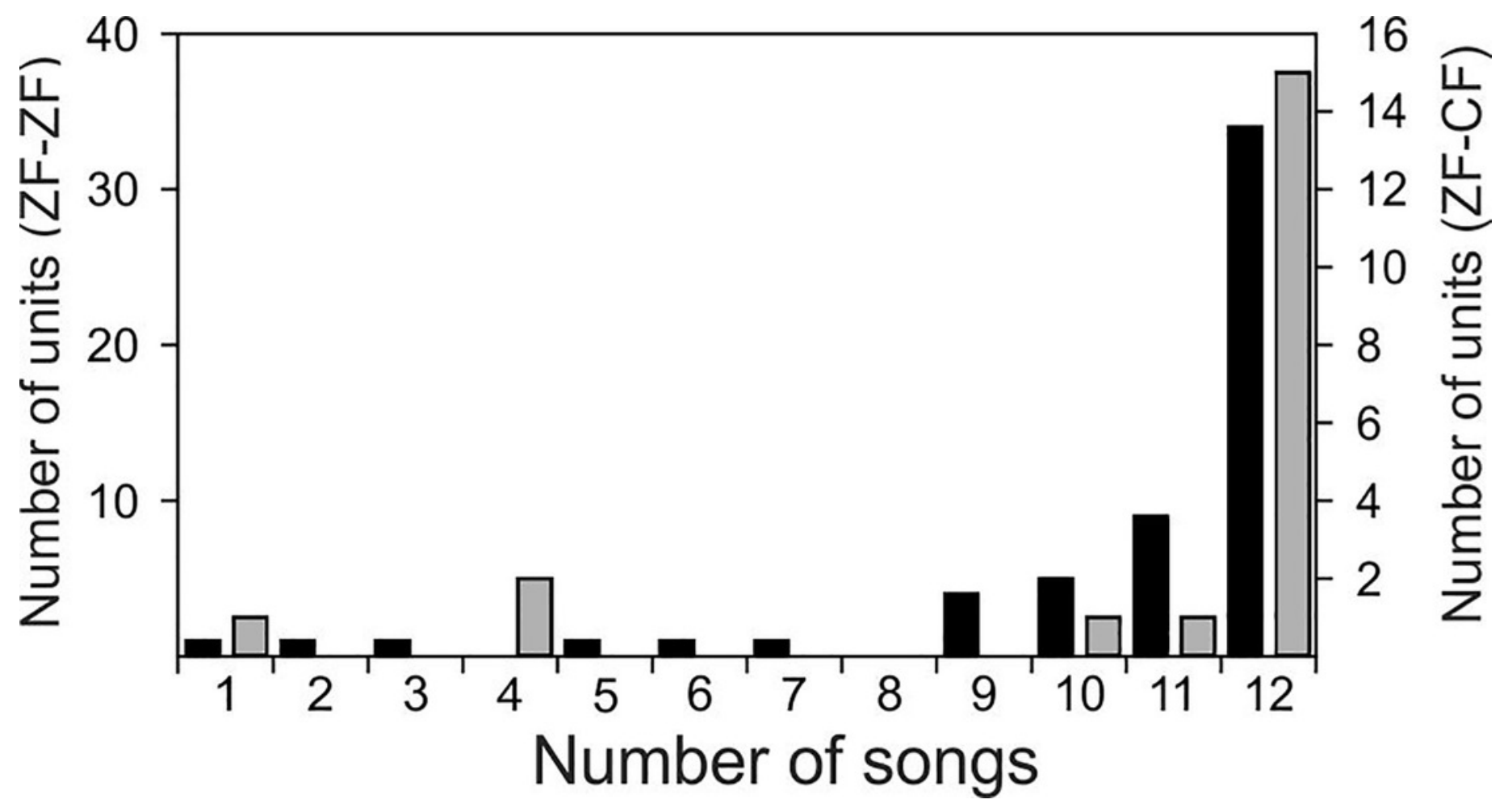




\section{Figure 5}

Examples of response patterns to song in MLd units from normal reared zebra finches (ZF-ZF)

The black arrows indicate the beginning of the sound stimulus and of the unit's response. (A) Example of a unit where the responses are locked to the AM envelope of the song and responded to most, if not all the components of the song. (B) Example of a unit responding with a sustained pattern throughout the presentation of the song. ( $C$ and $D$ ) Examples of the same unit's response to a conspecific and heterospecific song. In response to conspecific song (C) the unit shows a strong responses to two but not all harmonic stacks (grey arrows and white arrows respectively). In response to a heterospecific song that does not contain harmonic stacks, the same unit shows strong responses to the first series of trills, but not to the second half of the trills (white arrows). 

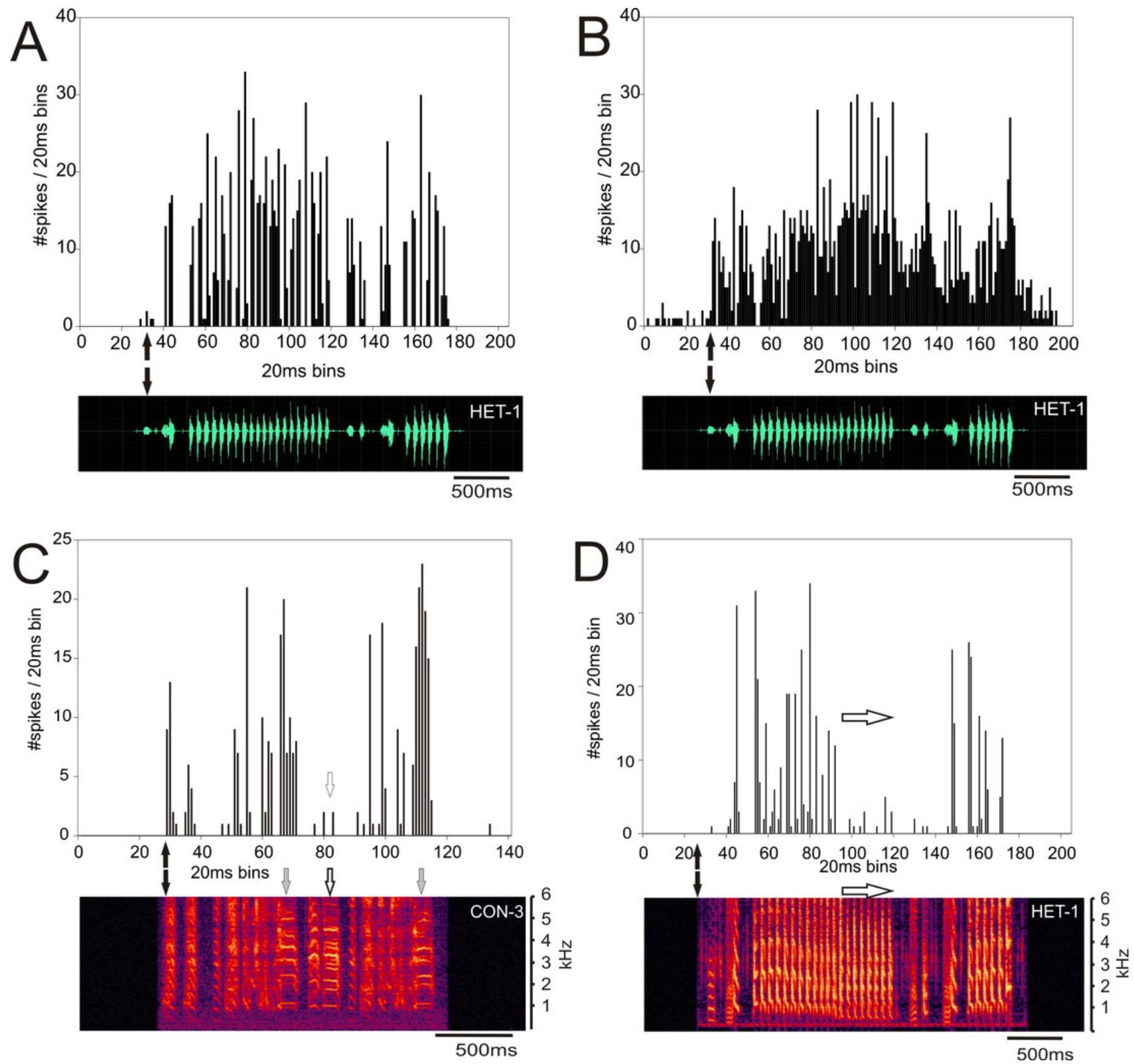


\section{Figure 6}

Inhibition and excitation by different stimuli in the same MLd unit of a cross-fostered zebra finch (ZF-CF)

The black arrows indicate the beginning of the sound stimulus and of the unit's response.

This unit showed inhibition in response to white noise $(A)$ and a conspecific song (B), but is excited with the heterospecific song.
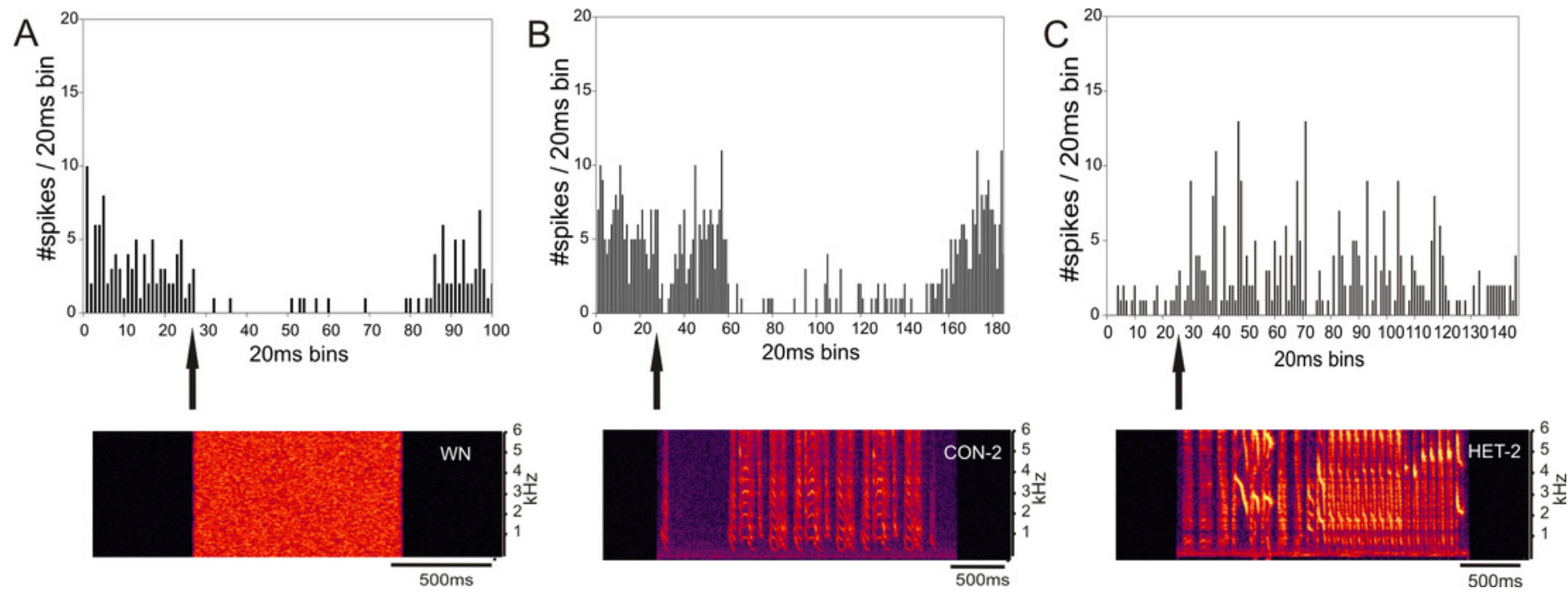


\section{Figure 7}

Cumulative distribution of $d^{\prime}$ values

(A) Cumulative distribution of d' values for the 58 individual MLd units recorded from in response to conspecific song 2 (CON-2) against its reverse version and all other forward song stimuli in normal reared zebra finches (ZF-ZF). (B) Cumulative distribution of d' values for the 20 individual MLd units recorded from for heterospecific song 1 (HET-1) against its reverse version and all other forward song stimuli in cross-fostered zebra finches (ZF-CF). (C) Cumulative distribution of $d$ ' values for the 58 individual MLd units recorded from in response to heterospecific song 1 (HET-1) against its reverse version and all other forward song stimuli in normal reared zebra finches (ZF-ZF). (D) Cumulative distribution of d' values for the 20 individual MLd units recorded from for conspecific song 2 (CON-2) against its reverse version and all other forward song stimuli in cross-fostered zebra finches (ZF-CF). In all plots blackfilled circles represent non-selective instances as determined by SI value and unfilled circles represent selective instances as determined by SI value (see Methods) The vertical black line delimit the non-selectivity zone where $d^{\prime}>|2|$. 

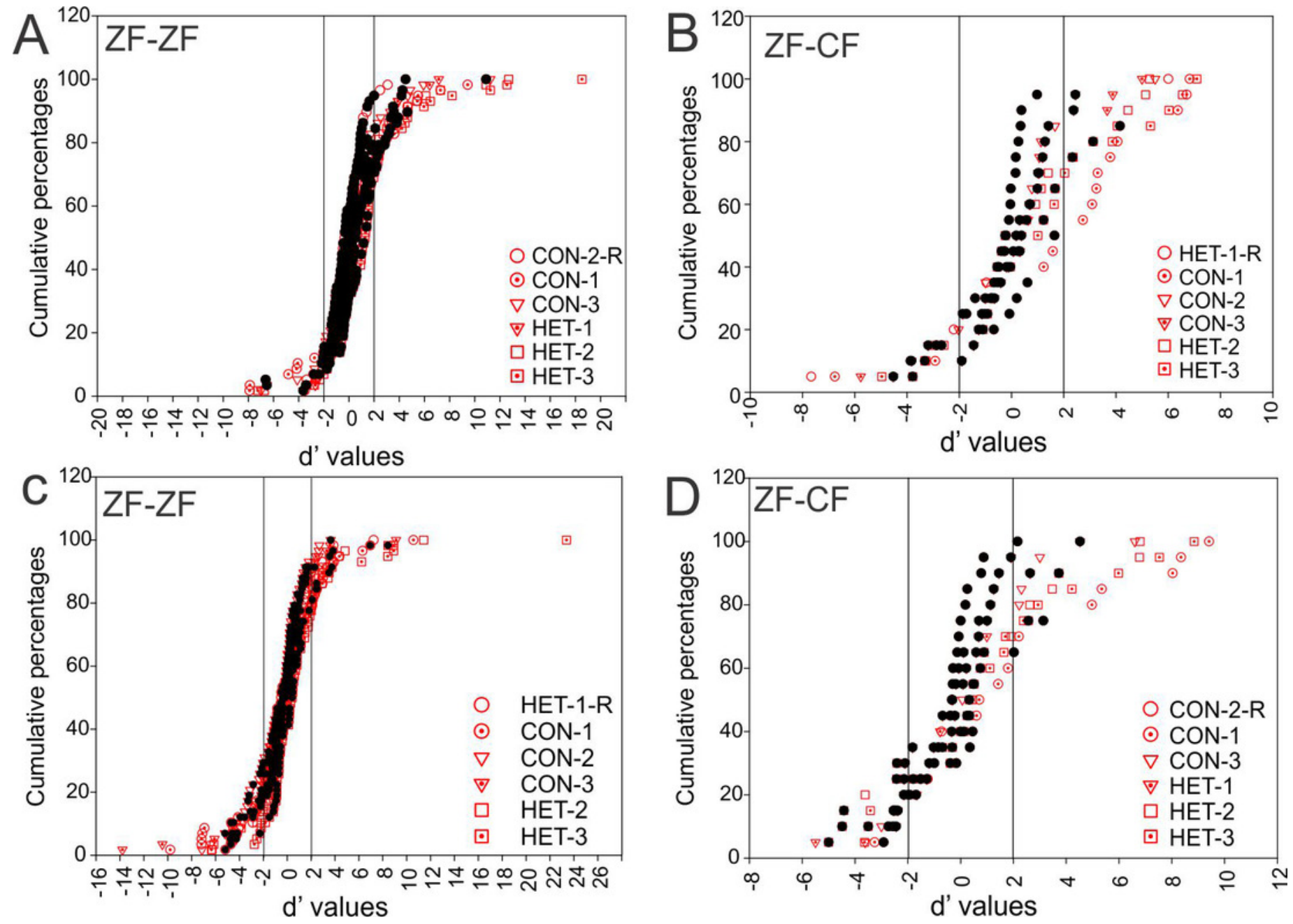


\section{Figure 8}

Distribution of $d^{\prime}$ values

(A) Distribution of d' values for 58 MLd units from normal reared zebra finches (ZF-ZF) for conspecific song 2 (CON-2) against other forward song stimuli.(B) Distribution of d' values for 20 MLd units from cross-fostered zebra finches (ZF-CF) for conspecific song 2 (CON-2) against all other forward song stimuli. (C) Distribution of d' values for 58 MLd units from normal reared zebra finches (ZF-ZF) for heterospecific song 1 (HET-1) against other forward song stimuli. (D) Distribution of d' values for 20 MLd units from cross-fostered zebra finches (ZFCF) for heterospecific song 1 (HET-1) against all other forward song stimuli. (In all plots the horizontal black lines delimit the non-selectivity zone where $d^{\prime}>|2|$. Data points above the non-selectivity zone indicate instances in which CON-2 ( $A$ and B) or HET-1 (C and D) were preferred over the other songs they were tested against. Data points below the nonselectivity zone indicate instances in which the other song stimuli were preferred over CON-2 (A and $B$ ) or HET-1 (C and D). 

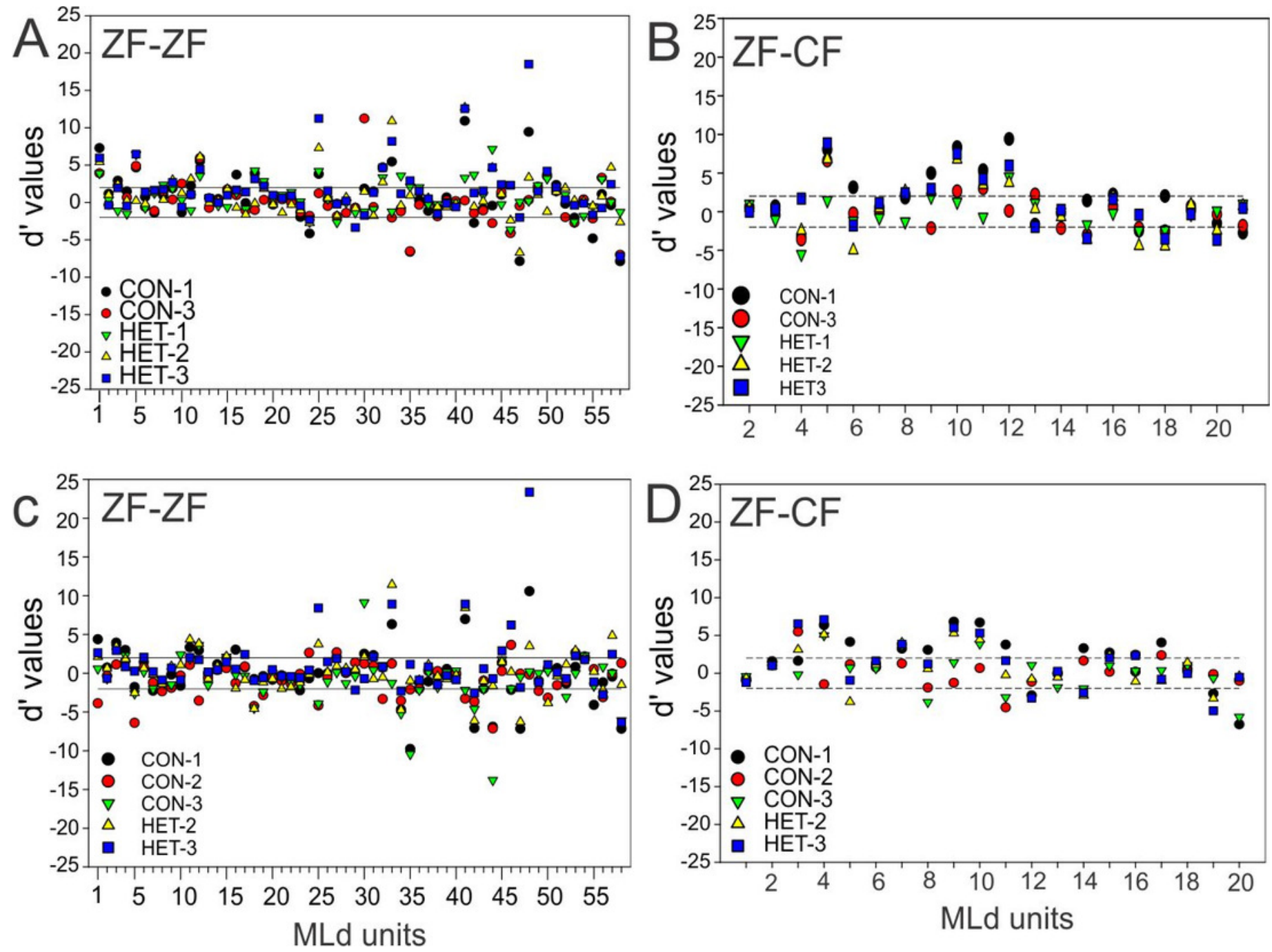
Figure 9

Distribution of RS values

(A) Distribution of RS values (spikes/sec) for all song stimuli presentations from MLd units from normal reared (ZF-ZF, black bars) and cross-fostered (ZF-CF, grey bars) zebra finches. (B) RS (spikes/sec) values for ZF-ZF (black) and ZF-CF (gray) for all units in response to conspecific (CON) or heterospecific (HET) stimuli. The range and mean of the responses are indicated for each group. 


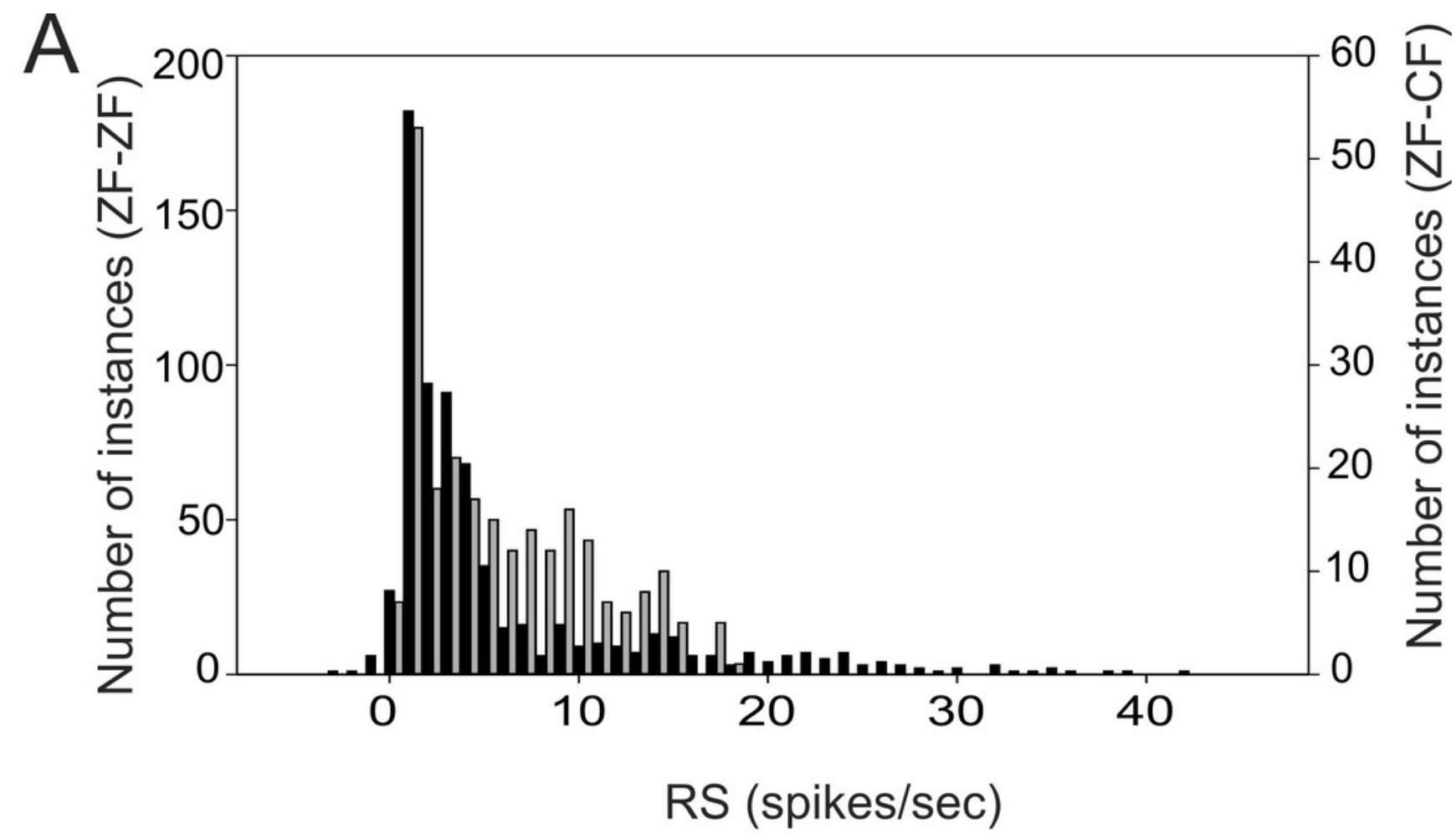

B

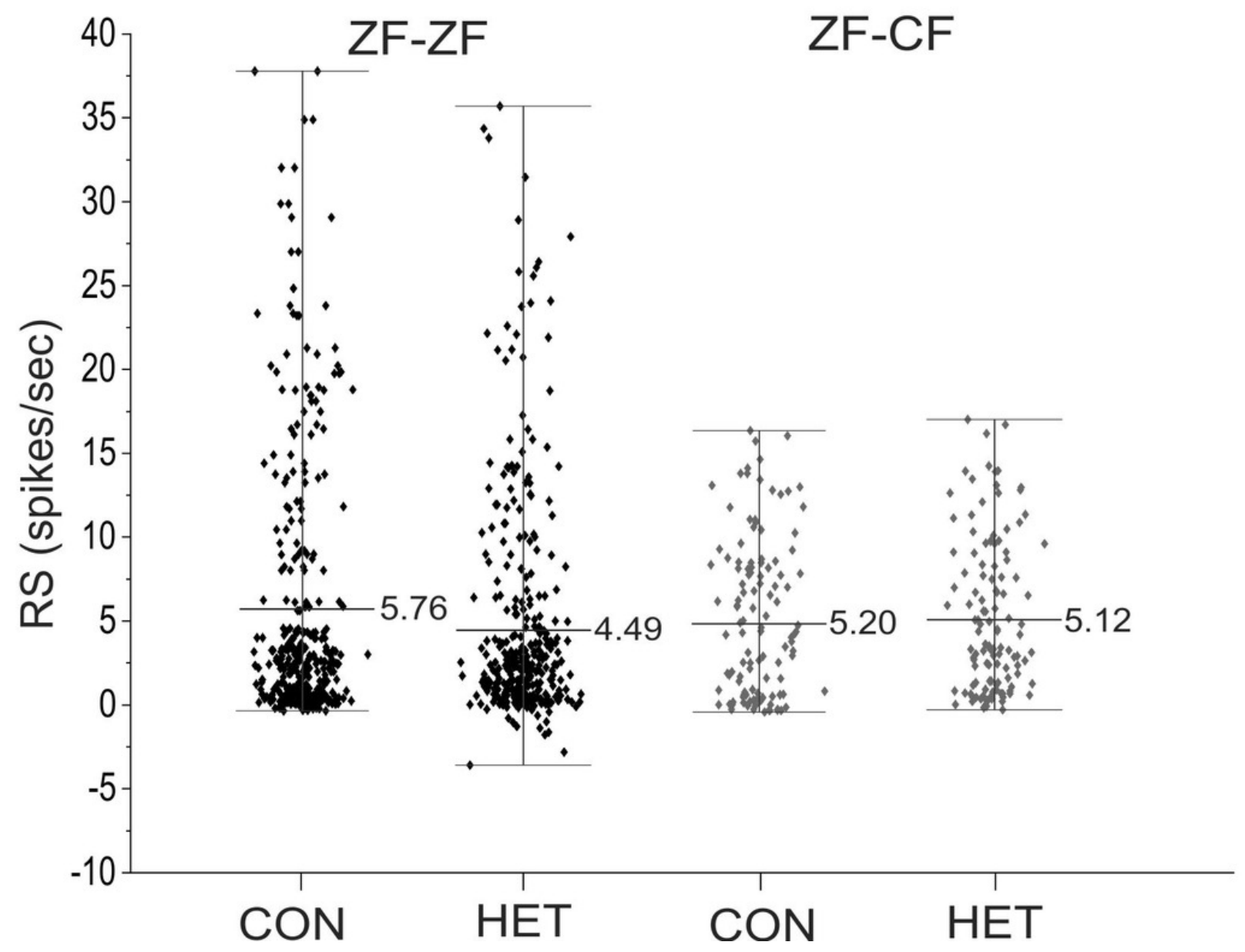




\section{Figure 10}

Mean RS value of individual units

Plots of mean RS values for each individual unit in response to a forward song vs. its reverse version. (A) normal reared zebra finches (ZF-ZF); (B) cross-fostered zebra finches (ZF-CF). In both plots the diagonal line indicates equal response to forward and reverse version. (C) and (D) Histograms showing the minimum distance of individual data points to the identity line calculated as: (RS to FOR version of the song + RS to REV version of the song)/ 2 . Positive values were assigned to instances where response to FOR song is larger than to REV song and negative values were assigned to instances where responses to REV song are larger than to FOR song. Percentages indicate the proportion of units that fall either above, or below the identity line.
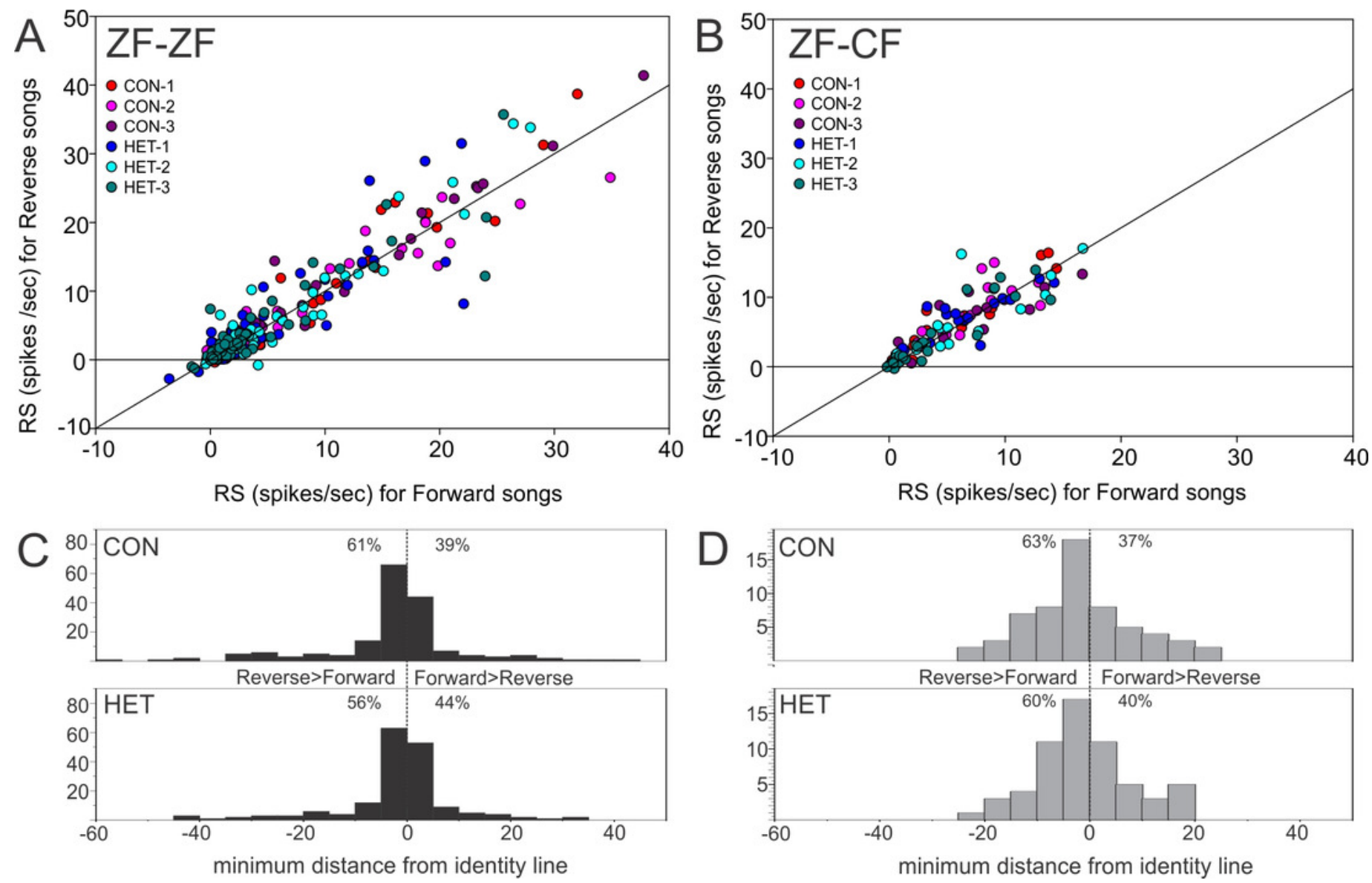


\section{Table $\mathbf{1}$ (on next page)}

Percentage (number) of units responding to $\mathrm{WN}$ and individual tones in normal reared (ZF-ZF) and cross fostered (ZF-CF) birds 
1 Table1: Percentage (number) of units responding to $\mathrm{WN}$ and individual tones in normal 2 reared (ZF-ZF) and cross fostered (ZF-CF) birds.

\begin{tabular}{|l|c|c|c|c|c|c|c|c|}
\hline & $\mathrm{WN}$ & $1 \mathrm{kHz}$ & $2 \mathrm{kHz}$ & $3 \mathrm{kHz}$ & $4 \mathrm{kHz}$ & $5 \mathrm{kHz}$ & $6 \mathrm{kHz}$ & none \\
\hline ZF-ZF & $86 \%(50)$ & $76 \%(44)$ & $72 \%(42)$ & $48 \%(28)$ & $24 \%(14)$ & $10 \%(6)$ & $2 \%(1)$ & $2 \%(1)$ \\
\hline ZF-CF & $80 \%(16)$ & $75 \%(15)$ & $85 \%(17)$ & $75 \%(15)$ & $15 \%(3)$ & $30 \%(6)$ & $5 \%(1)$ & $0 \%(0)$ \\
\hline
\end{tabular}

3

4 


\section{Table 2 (on next page)}

Percentage (number) of units with different BF in normal reared (ZF-ZF) and cross fostered (ZF-CF) birds. 
1 Table 2: Percentage (number) of units with different BF in normal reared (ZF-ZF) and cross 2 fostered (ZF-CF) birds.

\begin{tabular}{|l|c|c|c|c|c|c|}
\hline & $1 \mathrm{KHz}$ & $2 \mathrm{KHz}$ & $3 \mathrm{KHz}$ & $4 \mathrm{KHz}$ & $5 \mathrm{KHz}$ & $6 \mathrm{KHz}$ \\
\hline ZF-ZF & $26 \%(15)$ & $34 \%(20)$ & $24 \%(14)$ & $5 \%(3)$ & $2 \%(1)$ & $0 \%(0)$ \\
\hline ZF-CF & $35 \%(7)$ & $30 \%(6)$ & $25 \%(5)$ & $5 \%(1)$ & $5 \%(1)$ & $0 \%(0)$ \\
\hline
\end{tabular}

3

4 


\section{Table 3 (on next page)}

Responses to different types of stimuli by MLd units in ZF-ZF (58 units) and ZF-CF (20 units) 
1 Table 3: Responses to different types of stimuli by MLd units in ZF-ZF (58 units) and ZF-CF (20 2 units).

3

\begin{tabular}{|c|c|c|}
\hline & ZF-ZF & ZF-CF \\
\hline Responsive only to songs & $2 \%$ & $0 \%$ \\
\hline Responsive to tones and songs & $12 \%$ & $25 \%$ \\
\hline Responsive to WN and songs & $7 \%$ & $0 \%$ \\
\hline Responsive to all 3 stimuli & $79 \%$ & $75 \%$ \\
\hline Tones > WN & $52 \%$ & $20 \%$ \\
\hline WN $>$ Tones & $48 \%$ & $80 \%$ \\
\hline
\end{tabular}

4 
Table 4 (on next page)

Significant main effects and interactions as determined by the GLMM analysis 
1 Table 4: Significant main effects and interactions as determined by the GLMM analysis.

2

\begin{tabular}{|l|c|c|c|c|}
\hline \multicolumn{5}{|c|}{ Type 3 tests of fixed effects } \\
\hline Effect & Num DF & Den DF & F Value & Pr>F \\
\hline Individual song & 4 & 384 & 4.95 & 0.0007 \\
\hline Song direction & 1 & 467 & 16.87 & $<0.0001$ \\
\hline Rearing*CON/HET & 1 & 384 & 4.12 & 0.0429 \\
\hline
\end{tabular}

3 


\section{Table 5 (on next page)}

Least Squares Means 
1

Least

Squares

Means

\begin{tabular}{|l|l|l|l|l|l|l|l|l|l|}
\hline Effect & Category & $\begin{array}{l}\text { Song } \\
\text { ID }\end{array}$ & $\begin{array}{l}\text { Song } \\
\text { type }\end{array}$ & Direction & Estimate & $\begin{array}{l}\text { Standard } \\
\text { error }\end{array}$ & DF & T value & Pr $>|t|$ \\
\hline $\begin{array}{l}\text { Category*song } \\
\text { type }\end{array}$ & ZF-CF & & CON/HET & & 0.9706 & 0.05651 & 22.7 & 17.18 & $<0.0001$ \\
\hline $\begin{array}{l}\text { Category*song } \\
\text { type }\end{array}$ & ZF-ZF & & CON/HET & & 0.9479 & 0.04053 & 8.3 & 23.38 & $<0.0001$ \\
\hline Direction & & & & FOR/REV & 0.9407 & 0.03458 & 15 & 27.21 & $<0.0001$ \\
\hline Song(song type) & & $\begin{array}{l}\text { CON- } \\
1\end{array}$ & CON & & 0.9380 & 0.03551 & 16.8 & 26.41 & $<0.0001$ \\
\hline Song(song type) & & $\begin{array}{l}\text { CON- } \\
2\end{array}$ & CON & & 0.9679 & 0.03551 & 16.8 & 27.25 & $<0.0001$ \\
\hline Song(song type) & & $\begin{array}{l}\text { CON- } \\
3\end{array}$ & CON & & 0.9719 & 0.03551 & 16.8 & 27.37 & $<0.0001$ \\
\hline Song(song type) & & HET-1 & HET & & 0.9506 & 0.03551 & 16.8 & 26.77 & $<0.0001$ \\
\hline Song(song type) & & HET-2 & HET & & 0.9466 & 0.03551 & 16.8 & 26.65 & $<0.0001$ \\
\hline Song(song type) & & HET-3 & HET & & 0.9128 & 0.03551 & 16.8 & 25.70 & $<0.0001$ \\
\hline
\end{tabular}

